Boise State University

ScholarWorks

Materials Science and Engineering Faculty

Publications and Presentations

Department of Materials Science and Engineering

7-1-2017

Computationally Connecting Organic Photovoltaic Performance to Atomistic Arrangements and Bulk Morphology

Matthew L. Jones

Boise State University

Eric Jankowski

Boise State University 
This is an author-produced, peer-reviewed version of this article. The final, definitive version of this document can be found online at Molecular Simulation, published by Taylor and Francis. Copyright restrictions may apply. doi: 10.1080/08927022.2017.1296958

\title{
Computationally connecting organic photovoltaic performance to atomistic arrangements and bulk morphology
}

\author{
Matthew L. Jones and Eric Jankowski* \\ Micron School of Materials Science and Engineering, 1910 University Dr., Boise, ID, 83725 \\ (15 September 2016)
}

\begin{abstract}
Rationally designing roll-to-roll printed organic photovoltaics requires a fundamental understanding of active layer morphologies optimized for charge separation and transport, and which ingredients can be used to self-assemble those morphologies. In this review article we discuss advances in three areas of computational modeling that provide insight into active layer morphology and the charge transport properties that result. We explain the computational bottlenecks prohibiting atomistically-detailed simulations of device-scale active layers and the coarse-graining and hardware acceleration strategies for overcoming them. We review coarse-grained simulations of organic photovoltaic active layers and show that high throughput simulations of experimentally-relevant length scales are now accessible. We describe a new Python package diffractometer that permits grazing-incidence X-ray scattering patterns of simulated active layers to be compared against experiments. We explain the accurate calculation of charge-carrier mobilities from coarse-grained active layer morphologies by using atomistic backmapping, quantum chemical calculations, and kinetic Monte Carlo simulations. We employ these simulations to show that ordering of poly(3-hexylthiophene-2,5-diyl) explains a factor of 1000 improvement in charge mobility. In concert, we present a suite of computational tools enabling large-scale electronic properties of organic photovoltaics to be studied and screened for by molecular simulations.
\end{abstract}

\section{Introduction}

Organic photovoltaics (OPVs) offer a potentially low-cost, scalable, sustainable power generation solution that could realistically meet projected global energy demands if their efficiency can be improved $[1,2]$. OPV devices convert sunlight into electrical current through a cascade of physical processes that depends on the morphology of the active layer[2-7]. The active layer is typically a mixture of electron-donating molecules and electron-accepting molecules whose miscibility leads to the formation of ordered, disordered, and mixed morphologies to varying degrees. Many factors determine the overall power conversion efficiency (PCE) of an OPV device, but engineering the nanostructured morphology of the active layer represents an opportunity to vastly improve PCE because fundamental understanding of how to reliably manufacture optimal morphologies is lacking. Precisely placing the quadrillions of active layer molecules in yet-undiscovered optimal arrangements can only be practically realized through molecular self-assembly. In order to harness the active layer's morphology to maximize the performance of organic electronic devices, it is also vital to identify the links between the orientations and conformations of the molecules and the devicescale bulk performance. The tasks of understanding which active layer ingredients self-assemble favorable morphologies and understanding which morphologies are optimal for OPV performance can be advanced through the use of computer simulations. Computer simulations have become an essential tool for predicting morphologies of materials systems, providing an understanding of how custom-tailored physical properties can be determined through nanostructural engineering[8].

*Corresponding author. Email: ericjankowski@boisestate.edu 
Computational chemistry allows the electronic properties of an active layer to be calculated by way of the electronic structure calculations of each constituent molecule[9-14]. Combining computational chemistry with modeled morphologies offers a feasible path to inform the design of low-cost, high-efficiency OPVs.

The low-cost of OPVs derives from their mass-manufacturability, wherein solvated active layer ingredients are spread continuously onto a substrate. This roll-to-roll processing can be done at low temperatures and can incorporate electronic circuitry with recent advances in printing technology $[1,15,16]$. During roll-to-roll manufacturing, thermodynamic free energy minimization drives the molecular self-assembly of active layer ingredients into a nanostructured morphology responsible for converting light into electricity. This morphology matters for overall PCE, because the bound electron-hole pairs (excitons) created when light is absorbed by electron-donating species must contact an electron-accepting species shortly after being created before the charge pair recombines and the absorbed photon's potential contribution to current is lost. Once charges are separated at the donor-acceptor interface, there must exist conducting paths from the interface to each electrode in order for the charges to escape the active layer and contribute to generated current. Therefore, high PCE OPVs require active layer morphologies optimized for the separation of excitons into distinct charges and the subsequent transport of these charges out of the active layer via high charge mobility.

Optimal morphologies for high PCE are hypothesized to have features including low mixed amorphous regions of donors and acceptors[17] or hierarchically structured mixed regions[18], but are still not known for certain. In devices made with donor poly(3-hexylthiophene-2,5-diyl) (P3HT) and acceptor [6,6]-phenyl $\mathrm{C}_{61}$-butyric acid methyl ester (PCBM) it is shown that 4-fold enhancement in PCE results from 10-30nm domains of phase-separated P3HT and PCBM on a CuI surface compared to devices less-ordered films prepared on PEDOT:PSS surfaces[19]. Thermal and solvent annealing of OPV films has also demonstrated increased ordering correlating with increased PCE[20, 21]. This evidence suggests that thermodynamic self-assembly can be used to engineer the structure of OPV active layers, contingent on finding ingredients and conditions that are suited to assemble morphologies optimized for high PCE.

In this work we review the advances to computationally predicting the morphology of OPV active layers, the difficulties associated with understanding experimental and simulated active layer morphologies, the processes for mapping between molecular models with varying detail, and the computation of charge mobility from device-scale morphologies. These components comprise a framework of computational tools that can now be leveraged to advance fundamental understanding of active layer engineering. Charge mobility calculations permit the fundamental barriers to efficient charge transport to be elucidated for specific active layer mixtures, and for the mobilities of a set of morphologies to be directly compared. Coarse-grained molecular dynamics (MD) simulations permit device-scale morphologies to be efficiently predicted over broad regions of parameter space, allowing for the screening of active layer ingredients and conditions optimized to self-assemble target morphologies. Fine-graining methods that map coarse-grained morphologies back to atomistically-detailed representations permit charge mobility calculations to be applied to device-scale morphologies. Computational calculations of scattering patterns used to characterize OPV active layers permits simulated morphologies to be directly compared against experimental observations and provide insight into the molecular arrangements of realized OPV active layers. In concert, these computational capabilities hold promise to inform the engineering of low-cost, high PCE OPV's.

\section{Predicting morphology}

Simulations of materials with complexity comparable to OPVs are now routine, including systems of self-assembling surfactants, folding proteins, and sintering oxides[22-25]. Using molecular dy- 
namics (MD) and Monte Carlo (MC) techniques, the changing coordinates of the atoms comprising these systems can be evaluated to picometer $\left(1 \times 10^{-12} \mathrm{~m}\right)$ resolution. Simulations allow practical questions including "How does surface hydrophobicity influence nucleotide attraction?"[26], and simulations are essential to answering theoretical questions including "What is the densest packing of perfectly hard tetrahedra?" [27, 28].

Whether exploring the morphological possibilities for a particular mixture of ingredients or reverse-engineering which ingredients and processing conditions will self-assemble a target morphology, the utility of molecular simulations depend on their predictive capabilities. At the present time, molecular simulations are best suited to provide insights into the phase behavior of molecular mixtures as thermodynamic free energy minimization causes structures to evolve over time. These insights have been instrumental in painting a broad understanding of how self-assembly can be used to engineer active layer morphology, but additional advances are needed to fully characterize the nonequilibrium processes, boundary effects, and full time- and length-scales relevant to roll-to-roll OPV manufacturing. There are two key reasons modeling techniques need to to be improved to provide perfect information to OPV manufacturers: (1) It is prohibitively expensive to simulate the time-scales needed to observe atomistically-detailed self-assembly in 100-nm thick active layer films. (2) Nonequilibrium solvent evaporation, temperature gradients, and fluid flows that occurs during manufacturing are difficult to faithfully model with equilibrium simulation tools. In this work we focus discussion on MD simulations as the tool of choice for OPV morphology prediction, as MD better represents morphologies evolving over time compared to MC simulations that provide better insight into equilibrium properties at the cost employing unphysical atomic movements.

\subsection{Length vs. time trade-off}

One recent strategy for mitigating the computational cost of molecular simulations is to use computer hardware with the ability to perform expensive computations in parallel. Supercomputers are the standard way to leverage many processing units for parallelizeable computations including potential energy and force summations in a fraction of the time of a single processor. Recent advances in consumer gaming hardware has made available graphics processing units (GPUs) that act as small supercomputers, and have been leveraged to accelerate MD simulations to great success [29-36]. The speedups of MD packages including HOOMD-Blue, LAMMPS, and AMBER vary from $2 \mathrm{x}$ to $10 \mathrm{x}$, depending on the routines parallelized and implemented on GPUs and the systems used to perform benchmarks[29, 30, 33, 37]. Regardless of package used, the ability to increase by a factor of 2 to 10 the timescales accessible to a simulation by virtue of using GPU accelerators is expanding the utility of MD simulations for the study of OPVs and other materials. Due to these speedups and acceleration of other parallelizeable computations, GPUs or other massively multithreaded architectures are now an integral part of most supercomputer clusters.

Compounding the exponential computational cost of increasing system size is the fact that larger systems take longer to equilibrate, resulting in longer simulation times and limited accessibility of the equilibrium ensemble distribution of states. A striking example of the diminishing simulation timescales accessible in large simulations is apparent in the 2013 work of Carrillo et. al., which used the then-fastest GPU-accelerated supercomputer in the world (Titan) to simulate evolving morphology in P3HT:PCBM active layers[38]. Simulating nearly 4 million particles, this work accessed the $100 \mathrm{~nm}$ length-scales needed to model the full height of an active layer and accessed 400ns of simulation time, a significant achievement in scientific computing. However, if morphological evolution on the timescale of seconds is required to understand OPV active layers then the cost of a evaluating a single time step in this model needs to be lowered by a factor of 25 million. In the case of this 400ns simulation the potential energy had not converged to a stable average, indicating the equilibrium distribution of states had not been reached. The problem gets even worse when sampling equilibrium ensembles is desired. After a simulation has relaxed to equilibrium, the num- 
ber of timesteps between statistically independent snapshots also increases with system size. Using correlations in potential energy as a proxy for correlations in structure, it is not unusual to find that around ten million time steps must be evaluated to obtain two statistically independent molecular configurations in $N=50,000$ models[39, 40]. It is therefore crucial to identify the largest system size possible, given existing computational resources, that will permit the necessary timescales for sampling to be accessed.

\subsection{Nonequilibrium processing challenges}

Faithfully representing the nonequilibrium processes that occur during the manufacturing of a printed OPV active layer is important for making predictive models, but is challenging because MD simulations generally employ inherently equilibrium-focused techniques. Considerable theoretical and computational effort has gone into the development of MD integration schemes, thermostats, and barostats for sampling thermodynamic ensembles of surface-free atomic configurations with numerical stability[41-43]. Consequently, there are logistical and theoretical challenges to modeling solvent evaporation, fluid flows, and temperature gradients over the course of a MD simulation on the scales needed to capture all the physics of roll-to-roll manufacturing. Imposing temperature gradients is the most accessible of these, as the methods of Müller-Plathe or Ikeshoji et. al. could be adapted to a variety of geometries [44, 45]. In the case of fluid flows, imposing more than 1D shear fields is not straightforward using the unphysical momentum transfer methods used by Müller-Plathe to calculate shear viscosity[46]. Most difficult is modeling the evaporation of solvent from an active layer during processing, as this imposes both concentration gradients and changing particle numbers that are not straightforward to handle. For example, removing a solvent molecule from a MD simulation introduces voids in the simulation volume over timescales that are unphysical compared to the (currently) intractable problem of simulating the film as it equilibrates in contact with an atmospheric blend of oxygen, nitrogen, and argon.

\subsection{Large-scale morphology predictions}

Despite the inability to fully capture all the length, time, and nonequilibrium considerations of active layer processing, MD simulations have proved useful in providing insight into large-scale active layer morphology. These simulations have accessed experimentally relevant length scales and time scales by using simplified coarse-grained models that significantly reduce computational cost. Coarse-grained models represent collections of connected atoms with coarse-grained simulation elements (equivalently termed "beads" or "particles") that reproduce the net interactions of those atoms with other coarse particles[47-51]. Accurate coarse-grained force fields require the potentials of mean force to be thoughtfully calculated from more detailed simulations if the coarse models are to be predictive over broad regions of state space[50, 52-54]. Alternatively, qualitative coarse-grained potentials permit the properties of a material system to be probed in response to assumptions about the underlying physics[38-40, 55-57].

Work by Jankowski, Marsh, and Jayaraman [39, 40] used a coarse-grained model of P3HT and PCBM inspired by the models of Do, Huang, and Faller[55-57] to study the phase behavior of P3HT derivatives mixed with fullerene derivatives. These simulations demonstrated agreement with experimental characterization of the neat polymer and polymer:PCBM mixtures, efficient sampling of parameter spaces, and identification of new structures that could enhance charge transport. Solvents were handled implicitly in these works, their effects captured by effective attractions modulated by Lennard-Jones $\epsilon$ values describing the relative attractions between side-chain beads, backbone beads, and acceptor beads. The simulations were performed using HOOMD-Blue[33], which permitted around 200 combinations of chain architectures, solvent types, temperatures, and acceptor fractions to be equilibrated over 4 months using NVIDIA c2050 GPUs. These simula- 
tions probed morphologies on the scale of $10 \mathrm{~nm}$, sampled for up to $2 \mu \mathrm{s}$, permitted self-assembled morphologies to be directly compared against experimental data (Figure 1). With the help of computational diffraction techniques described in detail in the next section, it was shown that the architecture of polymer side chains (whether they alternate or are aligned) determines the thermodynamic stability of layered structures vs. cylinders, in agreement with experiments[58]. The ability to screen multiple polymer types over a broad parameter space efficiently was essential to this work because the disorder-order transition temperatures for each molecule were not known a priori and simulations of fewer than 100ns were generally not sufficient to identify thermodynamically stable structures.
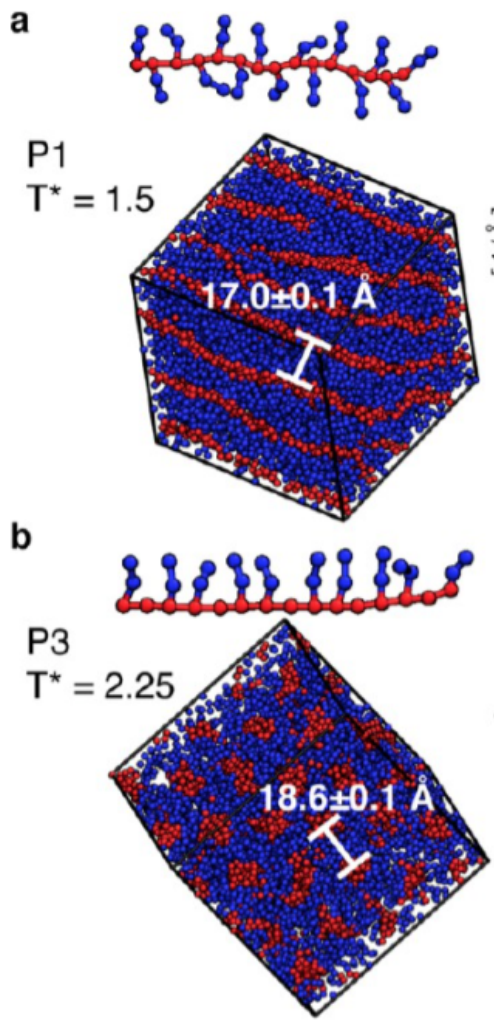
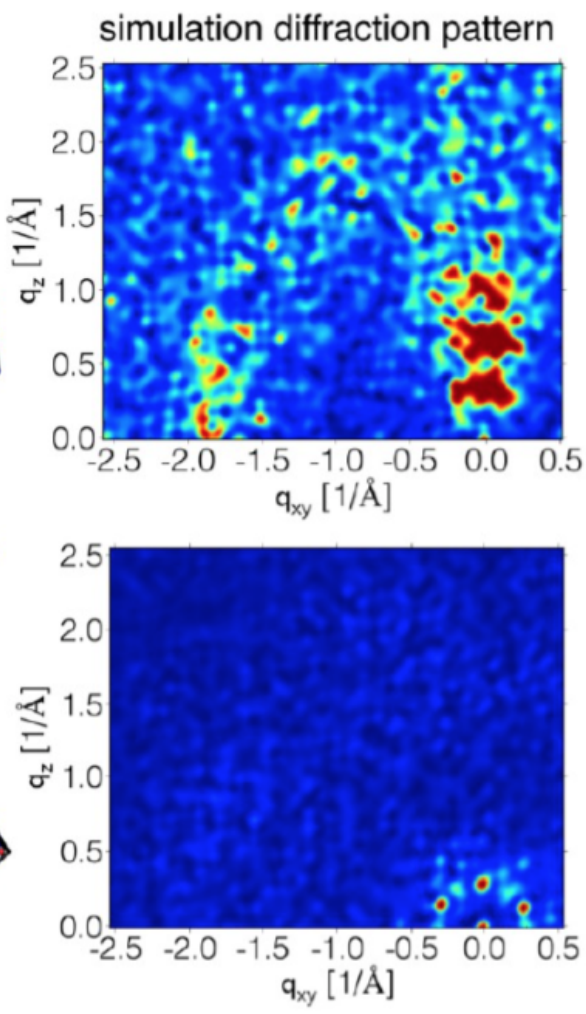
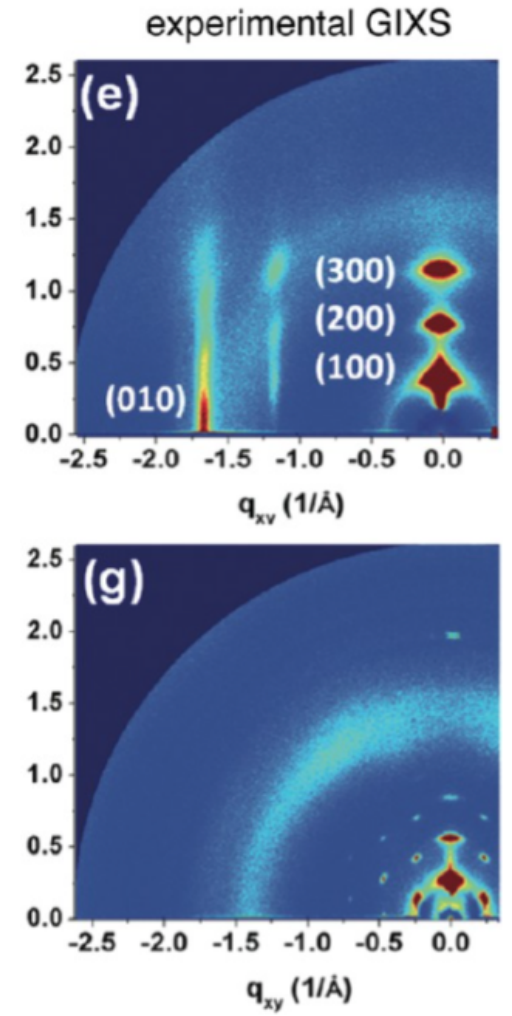

Figure 1. Coarse-grained simulations of neat P3HT and PDHBT access the length scales and timescales needed to reproduce experimental measurements. a) Imperfect lamellar structure assembled by model P3HT oligomers (left), corresponding diffraction pattern (center), and GIXS data from annealed P3HT (right). b) Hexagonally packed cylinders at assembled by model PDHBT (left), corresponding diffraction pattern (center), and GIXS data from annealed PDHBT (right). Reprinted with permission from Ref. [39]. Copyright 2013 American Chemical Society.

The utility of efficient screening of experimentally-relevant volumes was further demonstrated in Ref. [40], where the phase diagram of structure as a function of polymers of side chain type was elucidated ('a' = short length and strong attraction, 'b' = long length and strong attraction, 'c' = short length and weak attraction, 'd' = long length and weak attraction) (Figure 2)[40]. This work demonstrated that variations on chain length were sufficient to alter not only the disorder-order transition temperatures for the neat polymers, but also that lamellar, cylindrical, perforated lamellar, and ordered ribbon structures can all be tuned by the type of side chain. It also demonstrated that increasing side chain length increases the melting temperature of these polymers, in agreement with experiments performed in Ref. [59]. The ability to systematically vary side chain length and attraction strength without side effects was the key advantage in these exploratory simulations over atomistically-detailed models.

Once coarse-grained models are validated through comparisons with theory and experimental results, the ability to explore how changes in molecular geometry and miscibility influence morphology 


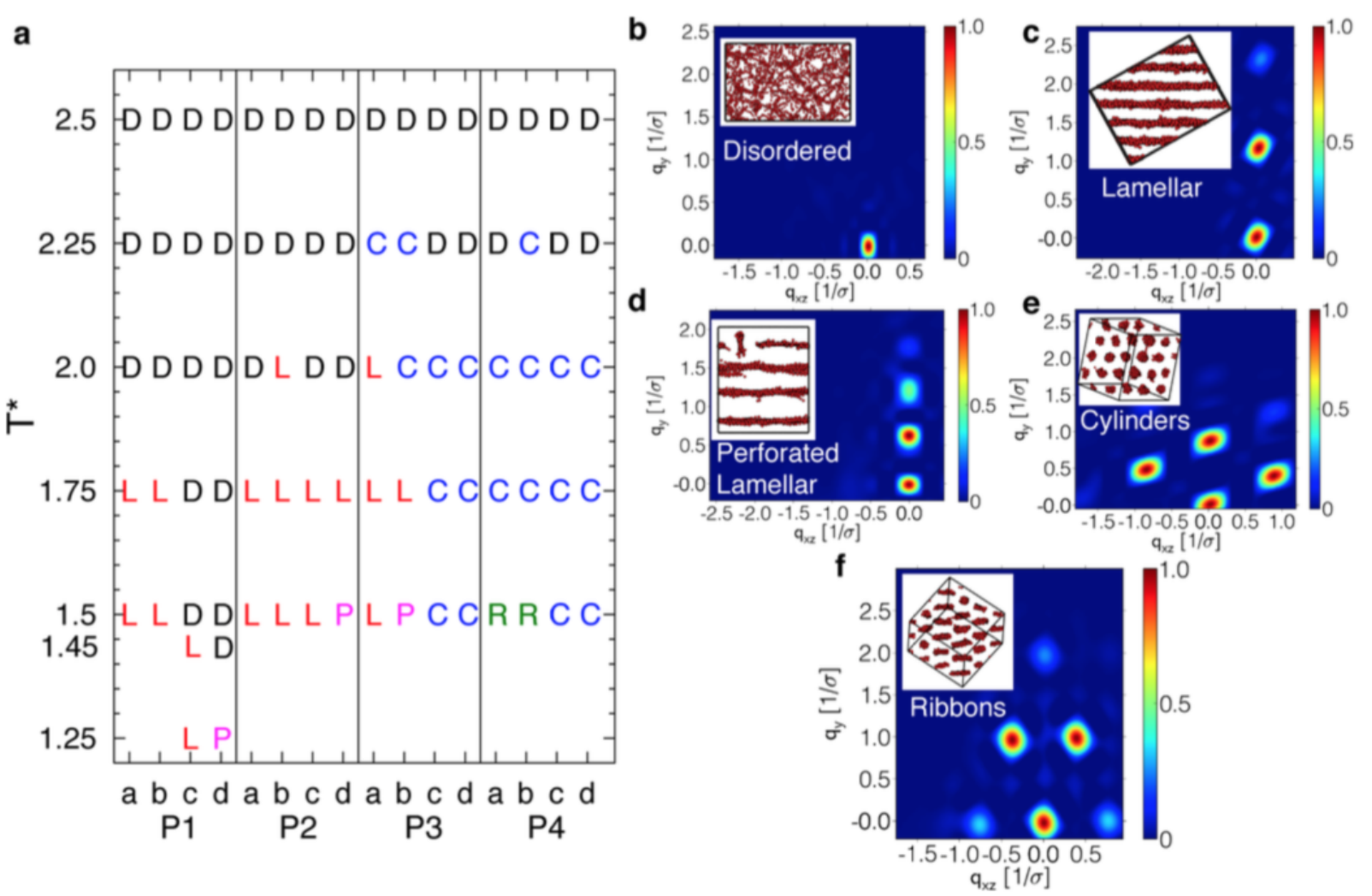

Figure 2. Sampling broad parameter spaces permits the role of side-chain length to be identified with coarse-grained models. a) Phase diagram of thermodynamically stable morphologies for for oligomer architectures P1, P2, P3, and P4 with side chain types a, b, c, and d. Representative diffraction patterns with snapshots inset for b) $\mathrm{D}=$ disordered, c) $\mathrm{L}=$ lamellar, d) $\mathrm{P}=$ perforated lamellar, e) $\mathrm{C}=$ cylindrical and f) $\mathrm{R}=$ ribbons. Reprinted with permission from Ref. [40]. Copyright 2014 American Chemical Society.

provides insight into design rules for guiding experiments. The simulations of model polythiophene derivatives mixed with fullerene derivatives in Ref. [39] were validated by both comparing neat P3HT and poly(3,4-dihexyl-2,2'-bithiophene) PDHBT morphology against experiments (Figure 1) and by comparing P3HT:PCBM and poly $\left(2,2^{\prime}: 5,22^{\prime \prime}-3,3\right.$ "-dihexylterthiophene) (PTTT):PCBM mixture morphology against experimental and theoretical predictions from Ref. [60]. In the former case, lamellar spacing and chain spacing of P3HT and the hexagonal packing of PDHBT was facilitated with simulated diffraction analysis. In the latter case, the intercalation of fullerenes in PTTT but not P3HT was shown to depend on the side-chain spacing. Having validated this coarse-grained model, Jankowski, Marsh, and Jayaraman performed simulations exploring how acceptor miscibility influenced the morphology of the mixtures (Figure 3 and Figure 4). A design rule resulting from this work was that the attraction between polymer backbones and fullerenes can be used to tune the thermodynamic stability of complex structures with high interfacial surface area between donors and acceptors. These structures include a kagome lattice of polymer backbones with acceptors aggregating at the triangular sites (Figure 4a) and cylinders of fullerenes surrounded by a sheath of polymer backbone (Figure 4c).

Using both GPU accelerators and coarse-grained models of OPV active layer ingredients, it is now possible to predict thermodynamically stable mixture morphologies efficiently. The morphologies resulting from these high-throughput simulations provide structural information that provides insight into detailed molecular arrangements and provides design rules for subsequent experiments. In the next section we discuss the details of simulated diffraction experiments that makes it possible to connect the predicted morphologies against experimental structures while helping to interpret experimental measurements. 

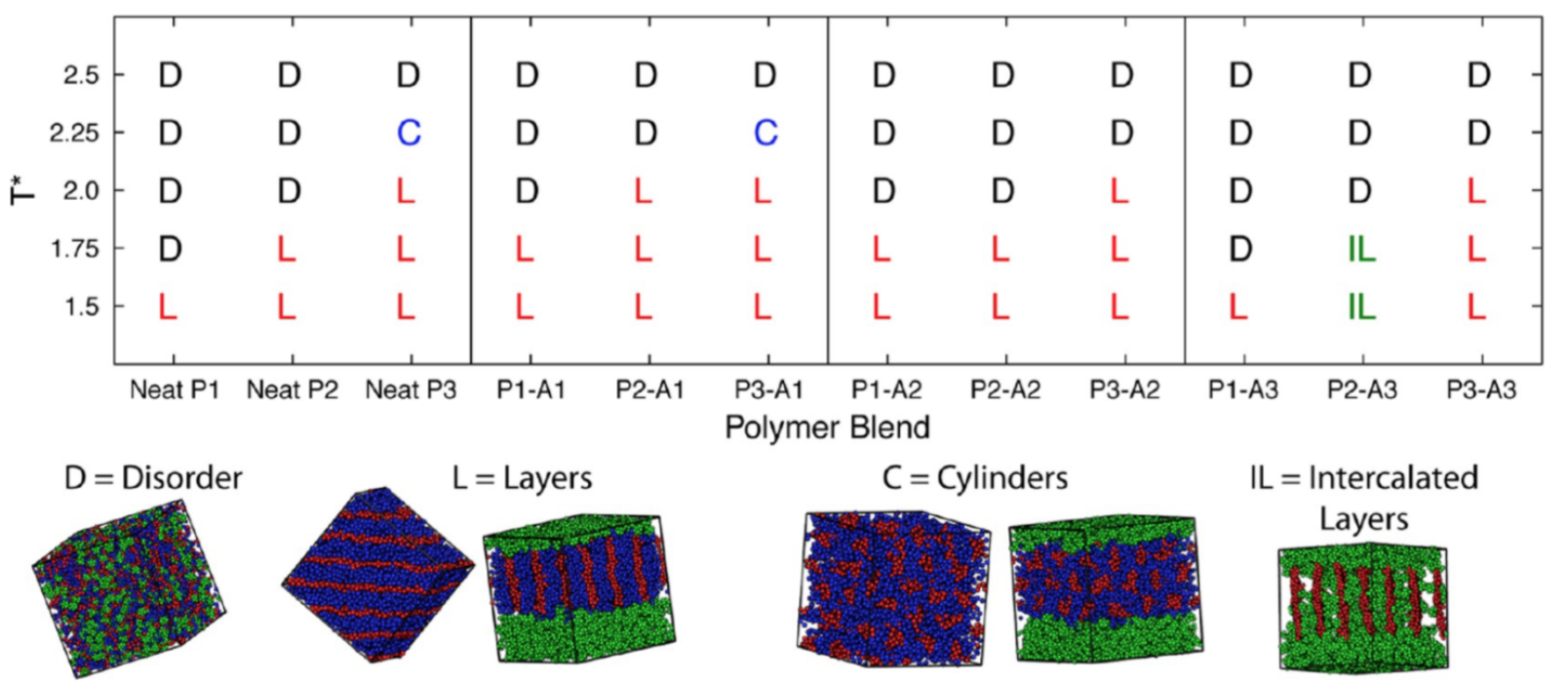

Figure 3. Phase diagram of thermodynamically stable morphologies for P3HT (P1), PTTT (P2) and PDHBT (P3) mixed with fullerene acceptors: A1 - strongly separates from the polymers, A2 - weakly miscible with polymers, and A3 - strongly miscible with polymers. Reprinted with permission from Ref. [39]. Copyright 2013 American Chemical Society.

\section{Characterizing morphology}

Given the growing importance of materials simulation, it may therefore come as a surprise that comparing simulated morphologies against their experimental counterparts is still not straightforward to do. The comparison challenge with OPV morphologies is caused by the nature of the indirect experimental scattering techniques used to probe active layer structure. These techniques measure the intensity of radiation scattered by a material as a function of the radiation's incident angle allowing determination of structural correlations within the material. The shortcoming of scattering techniques is that the scattering intensities are not unique-multiple arrangements of atoms can result in the same scattering pattern[61]. This is true whether the incident radiation is scattered by electron clouds (e.g., X-rays) or atomic nuclei (neutrons). The scattered intensities provide insight into the length scales of spatial correlations in a material, but these correlations are averaged over relatively large sample volumes, often masking non-negligible structural features[62]. Unfortunately, because of the scattering uniqueness problem, it is not generally possible to deconvolve a scattering pattern into atomic coordinates without additional information[61]. Therefore, the ability compare simulated structures against experimental ones depends on the ability to transform atomic coordinates into scattering patterns.

The challenges with inferring structure from scattering patterns are compounded in soft matter systems, where amorphous structures lacking symmetry or periodicity are both common and important. In the case of thin films made from organic polymers and fullerenes, complex morphologies that include multiple phases and varying degrees of order within one sample are commonly synthesized. Characterizing these films with grazing incidence X-ray scattering (GIXS) provides insight into structural correlations and their symmetries (if any), but insufficiently resolves the details of molecular packings in all but the most ordered structures[62]. In order to build a fundamental understanding of how the components and preparation of these films determines their morphology we require simulations that can reproduce experimentally realized morphologies that provide additional detail into their atomic arrangements. Consequently, we require the ability to generate scattering intensities of soft matter systems that can be compared with experiments.

Openly available code for performing scattering analysis exists for a variety of platforms. The MATLAB code accompanying Schmidt-Rohr's work permits scattering densities to be transformed into scattered intensities [63]. The ISAACS package by Le Roux and Petkov provides calculation 

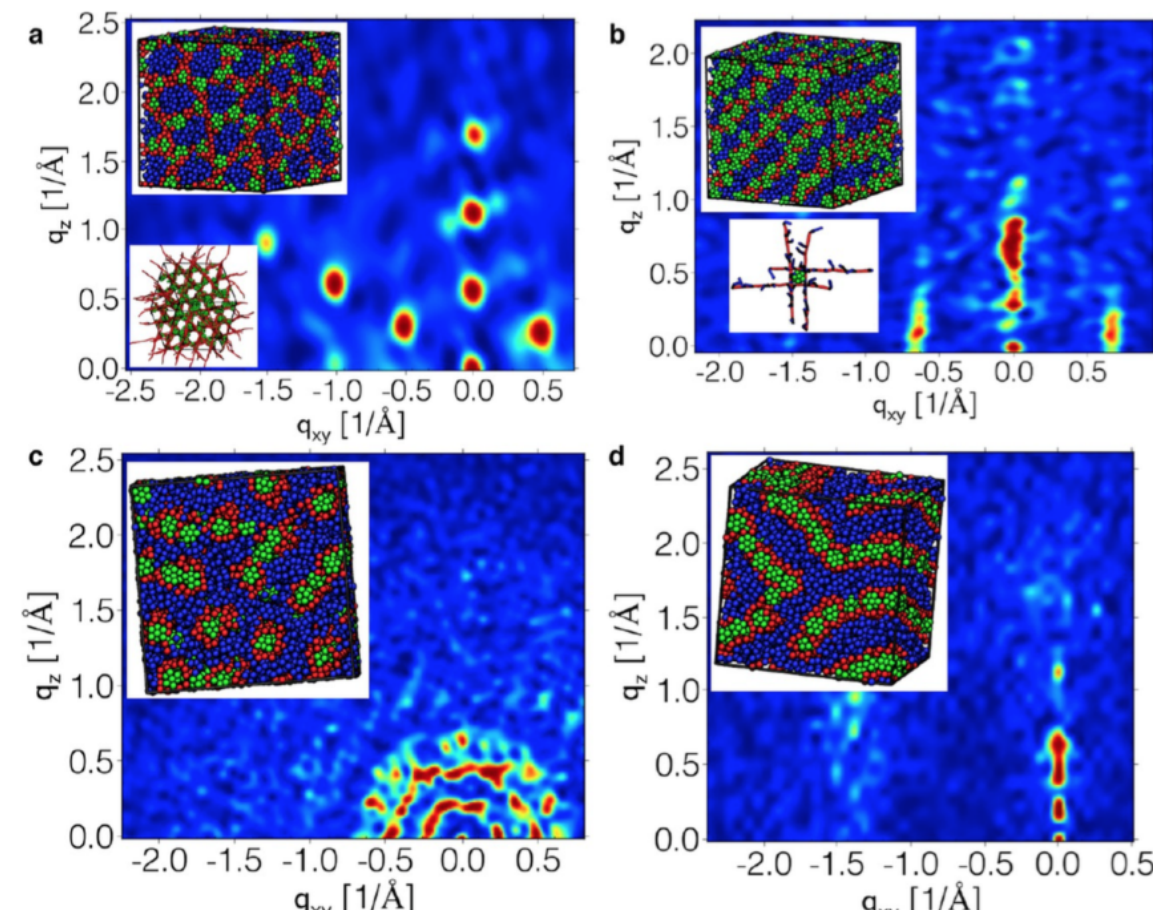

e
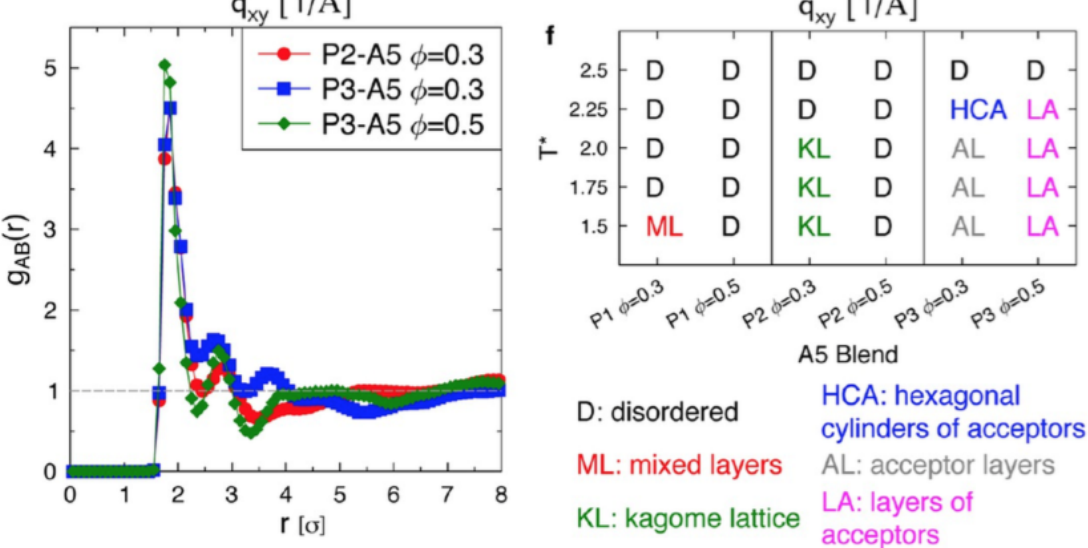

Figure 4. Exploratory simulations of fullerenes with strong attraction to the polymer backbone identify new structures with features that could enhance charge transport. Structural features of simulated OPV oligomers mixed with a fullerene derivative strongly attracted to the oligomer backbones. a) Kagome lattice of oligomers with fullerenes at triangular sites. b) Square lattice of oligomers intercalated in fullerene layers, c) Hexagonally-packed cylinders of fullerenes sheathed in oligomer backbones. d) Layers of fullerenes sheathed in oligomer backbones. e) Fullerene-backbone radial distribution functions quantifying increased acceptor-backbone correlations with increasing attraction. f) Phase diagram of strongly-attractive fullerene acceptor A5 with P3HT (P1), PTTT (P2) and PDHBT (P3). Reprinted with permission from Ref. [39]. Copyright 2013 American Chemical Society.

of structure factors with a variety of different techniques [64]. While the core mathematics to do so exists in both of these packages, neither provides a way to generate simulated GIXS patterns of arbitrary orientations of periodic simulation volumes easily.

Here we describe an open-source software package diffractometer for generating scattering patterns with a focus on soft matter systems. We review the scattering theory informing diffractometer, validate its correctness with scattering patterns of cubic crystals, and demonstrate its utility analyzing simulations of OPV films. 


\subsection{Scattering theory}

The scattered intensity $I(\vec{q})$ corresponding to a set of particle positions $\vec{x}$ depends on the density of scatterers in space $\rho(\vec{x})$. Specifically, the Fourier transform of the scattering density autocorrelation function $R_{\rho}(\vec{x})$ gives the scattered intensity[63]

$$
I(\vec{q})=\mathrm{FT}\left[R_{\rho}(\vec{x})\right] .
$$

The Fourier transform is defined by

$$
\operatorname{FT}\left[R_{\rho}(\vec{x})\right]=\int_{-\infty}^{\infty} R_{\rho}(\vec{x}) e^{-2 \pi i \vec{x} \vec{q}} d \vec{x},
$$

and the Inverse Fourier transform:

$$
\operatorname{IFT}[I(\vec{q})]=\int_{-\infty}^{\infty} I(\vec{q}) e^{2 \pi i \vec{q} \vec{x}} d \vec{q} .
$$

Autocorrelation functions such as $R_{\rho}(\vec{x})$ are convenient to calculate using Fourier transforms as well:

$$
R_{\rho}(\vec{x})=\operatorname{IFT}\left[\mathrm{FT}[\rho(\vec{x})] \mathrm{FT}^{*}[\rho(\vec{x})]\right],
$$

where $\mathrm{FT}^{*}[\rho(\vec{x})]$ denotes the complex conjugate of the Fourier transform of $\rho(\vec{x})$. Substituting Equation 4 into Equation 1, the scattered intensity simplifies to

$$
I(\vec{q})=\mathrm{FT}[\rho(\vec{x})] \mathrm{FT}^{*}[\rho(\vec{x})]=|\mathrm{FT}[\rho(\vec{x})]|^{2} .
$$

The result of Equation 5 provides the foundation for simulating scattering experiments: Given the scattering density $\rho(\vec{x})$ it is straightforward to calculate the scattered intensity $I(\vec{q})$ via Fourier transforms.

In order to generate the scattering density $\rho(\vec{x})$, upon which all these calculations depend, two components are needed. First, the positions of each scatterer in space $p(\vec{x})$. Second, the shape and orientation of each scatterer's scattering density $s(\vec{x})$. In the simple case of a system composed of identical scatterers, the scattering density can be calculated with

$$
\rho(\vec{x})=s(\vec{x}) * p(\vec{x})
$$

where $*$ is the convolution operator[63]. A consequence of Equation 6 and a Fourier transform property is that scattered intensities are efficient to calculate for systems of identical particles:

$$
\begin{gathered}
I(\vec{q})=|\mathrm{FT}[s(\vec{x}) * p(\vec{x})]|^{2} \\
I(\vec{q})=|\operatorname{FT}[s(\vec{x})] F T[p(\vec{x})]|^{2}
\end{gathered}
$$

Equation 8 permits high-efficiency calculations, because the multiplication of two matrices (here, performed in Fourier space) scales as the number of elements $O(N)$, whereas the convolution operation scales $O\left(N^{2}\right)$. In aggregate, using fast Fourier transforms that scale $O(N \log (N))$ followed by the $O(N)$ multiplication is faster than doing the $O\left(N^{2}\right)$ convolution followed by the $O(N \log (N))$ 
transformation. Another form of Equation 8 for isotropic systems is

$$
I(\vec{q})=\mathrm{FT}[\delta(r)+g(r)]\left|F T\left[\rho_{P}(\vec{x})\right]\right|^{2}=S(\vec{q}) P(\vec{q}),
$$

where $\rho_{P}$ is the density distribution of a spherical particle and $g(r)$ is the radial pair distribution function. This factorization is a convolution of the structure factor $S(\vec{q})=1+\rho F T[g(r)-1]$ and the form factor $P(\vec{q})=\left|F T\left[\rho_{P}(\vec{x})\right]\right|^{2}$. For isotropic fluids, the structure factor can be written

$$
S(\vec{q})=1+4 \pi \rho \int_{0}^{\infty} \frac{(g(r)-1) r^{2} \sin q r}{q r} d r
$$

and this form is frequently employed throughout the simulation literature. The problem with using Equation 10 in practice is that it will be inaccurate for anisotropic structures and the of different ways of replacing the upper infinite limit can give rise to a variety of artifacts[65].

It is preferable to use Equation 8 to directly calculate scattering intensities over Equation 10 because of its accuracy and also because it is increasingly straightforward to do so using numerical Fourier transforms made accessible through software including the Python numpy package. In the work of Schmidt-Rohr, calculation of the full 3D $I(\vec{q})$ is performed, which can subsequently be reduced to 2D GIXS or 1D structure factor representations[63]. One shortcoming of this approach is the large memory footprint of both the discretized 3D density distribution $\rho(\vec{x})$ and the corresponding intensity $I(\vec{q})$ field of a single configuration, which reach gigabytes each for typical systems. Another shortcoming is that it is difficult to efficiently implement the channel-sharing needed to generate 2D or 1D scattering intensities from the full 3D FFT. Here we demonstrate generation of $2 \mathrm{D}$ scattering intensities corresponding to GIXS patterns using discrete Fourier transforms of arbitrarily oriented periodic simulation volumes that overcomes the challenges of using a full 3D implementation.

\subsection{Computer implementation}

In order to determine the GIXS pattern corresponding to a scattering experiment from simulation data, two things are needed: (1) The scattering density throughout the periodic simulation volume, and (2) The orientation of the simulation volume with respect to the incident radiation. The latter is important because different orientations of an anisotropic simulation volume will generate different scattered intensities and require careful handling of periodic boundary conditions.

To explain the process of generating 2D GIXS patterns from simulation data, we consider an example case of a $4 \times 4 \times 4$ simple cubic lattice (Figure 5a) in a cubic simulation volume with box length $L=4$. The configuration shown in Figure $5 \mathrm{~b}$ is an example orientation of the cubic lattice for which the diffraction pattern is desired by applying arbitrary rotation matrix $R$ to the periodic volume. Applying Equation 8 to generate a GIXS pattern from the coordinates in Figure 5b, the projected 2D particle positions $p\left(\vec{x}_{x y}\right)$ and 2D single-particle scattering density $s\left(\vec{x}_{x y}\right)$ are needed as input to the 2D discrete Fourier transform. Here, $s\left(\vec{x}_{x y}\right)$ is taken to be a 2D Gaussian, which is its own Fourier transform pair. The positions $p\left(\vec{x}_{x y}\right)$ are obtained trivially from $p(\vec{x})$ by neglecting the $z$-coordinates of each particle in the rotated periodic cell. The projected coordinates require two transformations before the discrete Fourier transform can be applied: First, the $x$ and $y$ coordinates must be wrapped into a periodic area that tiles the plane. Second, that periodic area must be transformed (sheared) into a unit square so that particle positions may be discretized into a data structure that can be efficiently transformed into Fourier space. Any of the orthorhombically projected faces of the rotated unit cell will suffice as a periodic area in which to wrap projected coordinates. Here we use the largest of the three choices of face (front and back faces are equivalent, as are left and right, and top and bottom), shaded gray in Figure 5b. After applying periodic boundary conditions, the atoms outside the shaded gray area from Figure 5b are wrapped into 
it (Figure 5c). The shear matrix $M$ needed to transform the periodic area from Figure $5 \mathrm{c}$ to a unit square is determined by the inverse of the $2 \times 2$ matrix made from rotated box axes $a$ and $b$ bounding the shaded periodic area in Figure 5b:

$$
M=\left[\begin{array}{ll}
a_{x} & a_{y} \\
b_{x} & b_{y}
\end{array}\right]^{-1}
$$

The normalized coordinates $p^{*}\left(\vec{x}_{x} y\right)$ (see Figure $5 \mathrm{~d}$ ) are obtained using $M$ :

$$
p^{*}\left(\vec{x}_{x y}\right)=p\left(\vec{x}_{x y}\right) \cdot M^{T}
$$

where each row of matrix $p$ stores the $x$ and $y$ coordinates of the rotated atoms. Atom counts are then binned in an $N \times N$ matrix that serves as input to the discrete Fourier transform. Figure 5d shows $N=16$, and we find $N=512$ to be adequate for general-purpose use. After applying the uniform scattering density (here, a Gaussian blur) and resolving the intensity spectrum in Fourier space (Equation 8), the inverse shear matrix $M^{-1}$ is applied to the resulting diffraction pattern and the $\log _{10}$ of the bin intensities is calculated to give the final GIXS data (Figure 5e). The larger length-scale features can be "zoomed in" on by using fewer $N / z \times N / z$ discrete bins and then interpolating the $N \times N$ diffraction pattern from the final GIXS data. Physical wave vector $q$ axes are added to the final GIXS data (Figure 5f) using

$$
q\left(d_{p}\right)=\frac{2 \pi d_{p}}{L z}
$$

where $d_{p}$ is the distance in pixels of a GIXS bin from the origin and $L$ is the periodic box length. The code implementing these transformations is available online at http://bitbucket.com/cmelab/ cme_utils. Validation and verification details for our GIXS calculations are included in the supplementary information (SI section $\S 1$ ).

\section{Fine-Graining}

We refer to the process of mapping atomistic coordinates into a coarse-grained model as "finegraining", which is has found extensive use in the investigation of complex proteins[66-68], as well as other molecular systems[69-73]. Here, we use fine-graining to inform electronic property calculations, which complement the aforementioned structural analysis. Quantum chemical calculations (QCCs), such as density functional theory, can be used to to calculate important electronic properties for a molecular system such as the ground-state electronic structure and molecular orbital energies[9-14]. These calculations are therefore a prerequisite for quantifying the charge transport characteristics of a system. Unfortunately, in coarse-grained simulations, much of the electronic information is abstracted away, and so there exists no quantum chemical methodology that can operate on, or predict the electronic structure of, an arbitrarily coarse-grained molecule. As such, most studies that attempt to elucidate the links between the molecular morphology and bulk device performance are limited to atomistic representations of molecules, restricting the system size and relaxation timescales that can be simulated[56, 74-76]. Some recent investigations, however, have leveraged the computational efficiency of course-graining, combined with a 'fine-graining' or 'backmapping' process to return molecules to their atomistic representations for subsequent electronic structure calculations [66, 77-80].

Fine-graining can be implemented in several ways, but generally follows the same fundamental procedure, as depicted in Figure 6. The process of fine-graining maps atomistic coordinates onto coarse-grained simulation sites by taking one configuration (microstate) and projecting the atoms 

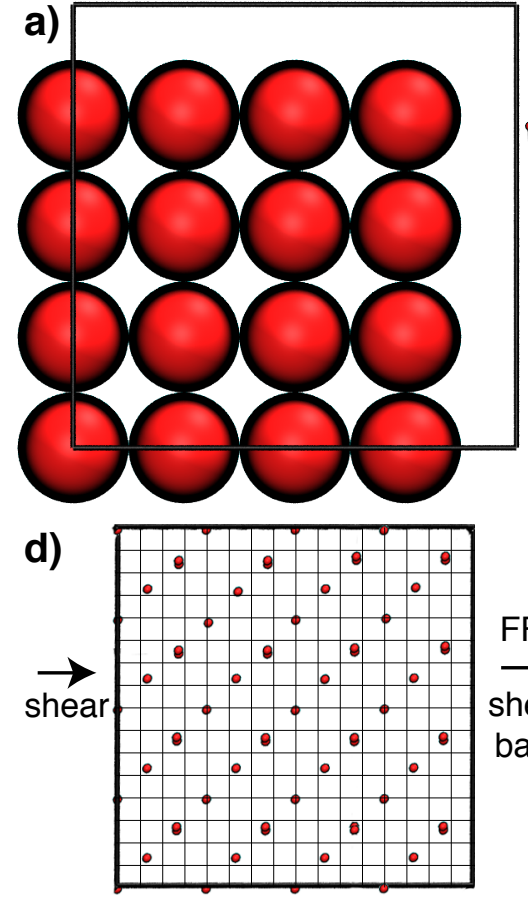


Figure 5. Example generation of an arbitrarily oriented simple cubic lattice diffraction pattern. a) 100 view of a $4 \times 4 \times 4$ simple cubic lattice of $1 \AA$ diameter spheres in a periodic simulation volume. b) An arbitrary orientation of the simple cubic lattice with the largest periodic face shaded gray. Only sphere centers are rendered for clarity. c) The resulting particle positions on the shaded gray periodic rhombus from (b) after all particles are projected onto the plane and periodic boundary conditions are applied. d) A shear transformation is applied to (c) to map the periodic rhombus onto a unit square. This square is discretized (16x16 shown, 512x512 used in practice) and the intensity value of a cell is incremented for each atomic center within it. e) The raw diffraction pattern corresponding to (d), generated after discrete Fourier transform application, normalization, and inverse shear transformation with the origin centered in the middle of the image. f) GIXS pattern with wave vector axes added.

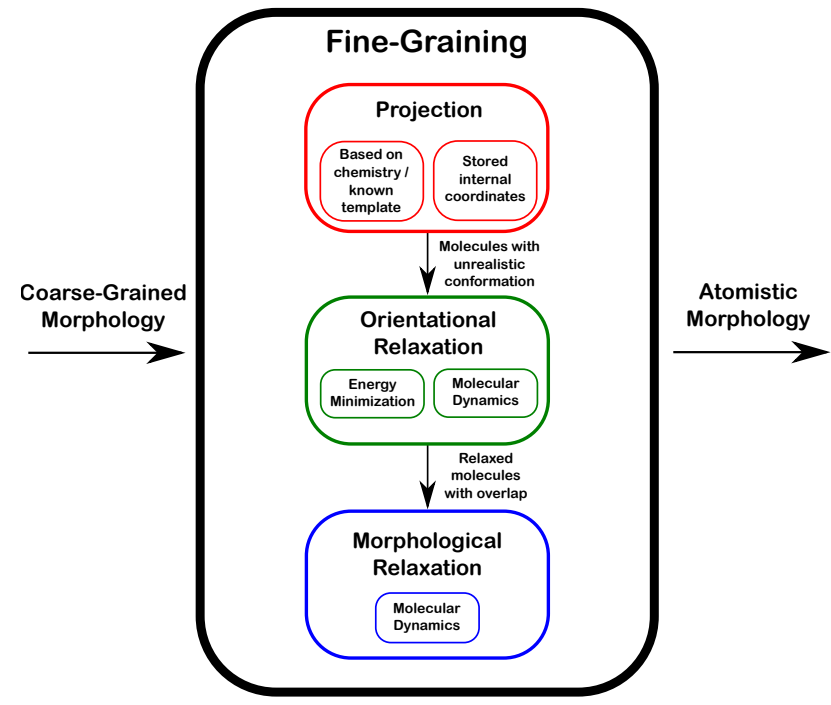

Figure 6. A scheme showing the three, basic requirements of a suitable fine-graining methodology, along with examples of the techniques frequently used to achieve each stage.

represented by each coarse-grained "bead". This mapping is constrained so the center-of-mass of the group of atoms is identical to the center-of-mass of the coarse-grained site. Subsequent relaxation of bonded constriants is required for each molecule to find a realistic atomistic morphology [69, 71], unless an internal coordiante system is maintained to permit constrains such as the expected tor- 
sional rotation of a thiophene ring or a phenyl group [70, 80] to be enforced during fine-graining. In some cases, it can be useful to treat each group as a rigid body, keeping constant the relative positions and orientations of atoms within the group according to a geometrically-relaxed template $[81,82]$. After the initial projection has been completed, there may be several unphysical molecular conformations - particularly for long, flexible polymer chains where backbones may wrap around and result in overlapping atoms. The atomic positions are therefore adjusted to make the conformation of the molecule more realistic, by energetically relaxing the molecules over very short timescales. This can be performed on a molecule-by-molecule basis in isolation $[67,68]$, or in situ using energetic minimization methodologies [69-71], or even full molecular dynamics simulations [72]. In some cases, particularly when polymer chains with high conformational disorder are being simulated, it is useful to apply additional geometrical constraints during the fine-graining process to maintain the original centers-of-mass of each group according to the fully equilibrated, corasegrained morphology generation. This can be achieved by simulating harmonic bonds with strong spring constants and small equilibrium lengths between the average positions of the atoms and the corresponding coarse-grained site position [72], anchoring the functional group in place. Such constraints can also help to simulate non-equilibrium effects such as a significant shear force [83]. A final relaxation stage is frequently performed with a longer molecular dynamics simulation, in order to fully relax the chains in the simulation volume and ensure that there are no atomic overlaps between molecules [69-72]. When a physically realistic, atomistic conformation for the molecule has been achieved, it can be used by QCCs to determine the electronic structure.



b)

c)
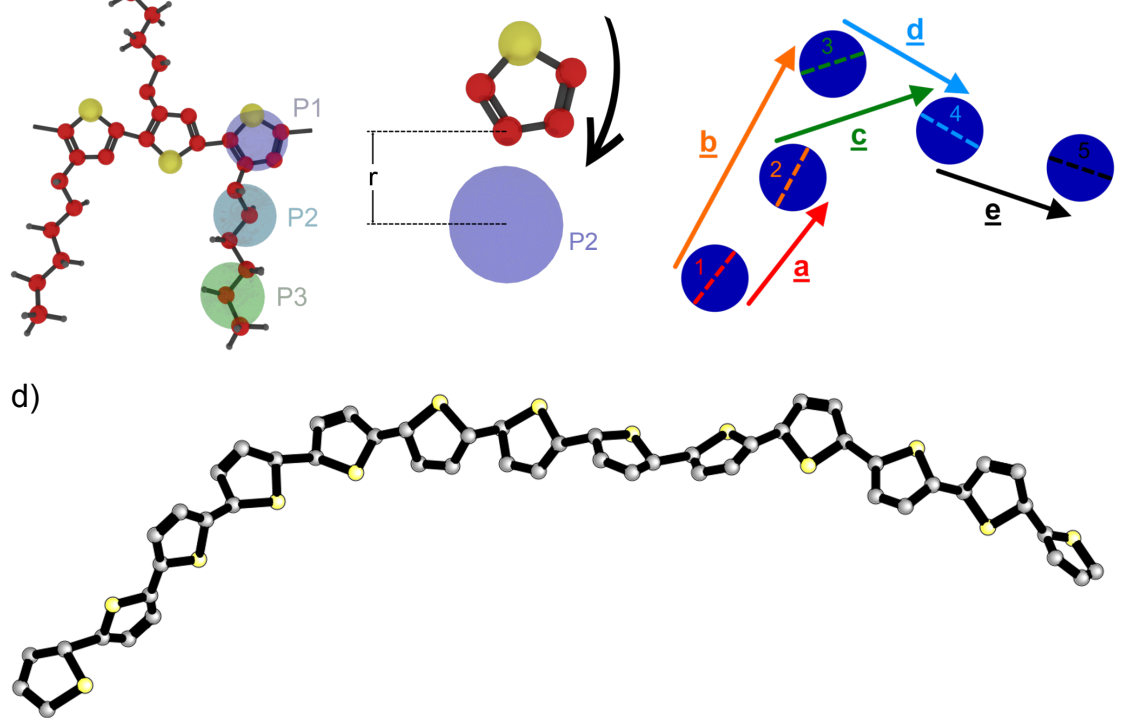

Figure 7. a) The coarse-grained model used to simulate P3HT. The sites P1, P2 and P 3 are located at the centers-of-mass of the thiophene ring, first three methyl group of the hexyl sidechain and the second three methyl groups respectively. b) The first rotation required to complete the fine-graining process of the P3HT morphologies, which flips the thiophene ring into the correct head-to-tail orientation to preserve regioregularity. c) The second rotation, which uses the locations of nearby monomers to determine the optimal ring planarity. d) The resultant conformation of a correctly rotated polythiophene backbone (P3HT without sidechains).

Determining a physical conformation for the initial projection of atoms is a non-trivial problem, due to the loss of rotational information during the process of coarse-graining into a single spherical bead. For instance, Figure 7a shows a commonly used, 3-site coarse-graining scheme for the example case of P3HT[39, 40, 56, 80]. The 24-atom monomer is mapped onto three coarse-grained beads, P1, P2, and P3, which represent the thiophene ring, and the first and second halves of the hexyl sidechain respectively. Given a particular center-of-mass for the site $P 1$, there are an infinite 
number of possible orientations for the plane of the thiophene ring it represents. However, due to the constraints caused by nearby bonded and non-bonded atoms, only a small quantity of these orientations are physically appropriate for the ring. These physical conformations can be estimated by considering the positions of nearby atoms - for example, by rotating the ring to minimize the distance between the center of mass of the bonded alkyl sidechain and the connecting carbon atom in the ring, as shown in Figure 7b. This has the effect of flipping the ring into the correct headto-tail orientation to maintain the original regioregularity of the chain. Minimizing this distance is an approximation designed to select an appropriate in-plane rotation of the ring, however, it does not take into account the bending of the sidechain due to the rotation of the ring along the chain's backbone axis, so the resultant conformation may not be energetically minimized. Therefore, this approximation can be improved by also considering the adjacent, bonded thiophene rings along the chain backbone.

One such minimal energy conformation along the backbone can be realized if the in-plane vector of a ring describes the vector between the two neighboring rings, as shown in figure Figure 7c. For instance, the plane of ring 2 in Figure 7 c can be matched to the vector $\underline{\mathbf{b}}$, which describes the relative positions of rings 1 and 3. This can be achieved by mapping the current normal vector of the ring, $\vec{a}$, to a desired target vector $\vec{b}$, by applying the shortest-possible-rotation matrix, $R$, to each atom's position:

$$
R=I+[v]_{\times}+[v]_{\times}^{2} \frac{1-(\vec{a} \cdot \vec{b})}{(|\vec{a} \times \vec{b}|)^{2}},
$$

where $I$ is the three-dimensional identity matrix, and $[v]_{\times}$is the skew-symmetric cross-product matrix of $v=\vec{a} \times \vec{b}=\left(v_{x}, v_{y}, v_{z}\right)$ :

$$
[v]_{\times} \equiv\left[\begin{array}{ccc}
0 & -v_{z} & v_{y} \\
v_{z} & 0 & -v_{x} \\
-v_{y} & v_{x} & 0
\end{array}\right]
$$

The shortest-possible-rotation ensures that the ring's head-to-tail orientation is not modified, maintaining the regioregularity of the chain selected by the algorithm depicted in Figure 7b. In the event that there are not three adjacently bonded thiophene rings (i.e. at the end of the chain as for thiophene ring 1 in Figure 7c), then the plane of the ring can be estimated as the vector to the single bonded neighbor (ring 2 , described by the vector $\underline{\mathbf{a}}$ ).

Although this can suitably orient the thiophene rings in the backbone (as shown in Figure 7d), the orientation of the alkyl sidechain has not yet been selected. Determining the conformation of the sidechain based purely on the positions of the three coarse-grained sites in each monomer is difficult because the sidechain can compress and extend significantly according to the carbon-tocarbon bonds along the sidechain's length. Additionally, as each molecule is fine-grained effectively in isolation with no consideration of nearby chains, the final simulation morphology is likely to contain a large number of overlapping atoms, which would prevent QCCs from producing accurate electronic structure results. Previous investigations have shown that the solubilizing sidechains do not affect the electronic structure of the molecule itself, instead only affecting the molecules conformation during the molecular dynamics relaxation process[84-86]. As the sidechains are included in the coarse-grained simulations, where they influenced the final conformation of the chain backbone, they can safely be omitted from the fine-graining process and the subsequent electronic structure calculations. This therefore treats the electronic structure of P3HT as identical to that of polythiophene. While this can provide good qualitative agreement with experimental mobility trends, it could contribute to the significant quantitative discrepancy sometimes observed[80]. An additional restriction is that this technique is strongly dependent on the molecule simulated, mak- 
ing it unsuitable for fine-graining other materials, where it may not be sufficient or appropriate to obtain rotation information based solely on the positions of the bonded coarse-grained sites.

A more robust method of fine-graining, which is transferable to other materials systems and can predict more accurate charge transport characteristics, leverages atomistic molecular dynamics simulations, optimized to run on GPUs, to find an acceptable relaxed conformation for all the molecules in the system simultaneously. The initial atomistic projection is done by crudely placing the moieties over the coarse-grained centers-of-mass with arbitrary rotations. In the example of P3HT, this results in a wide array of unphysical conformations, with elongated bonds between atoms, thiophene rings twisted and many atoms overlapping within the simulation volume, as shown in Figure 8a. Canonical NVT molecular dynamics simulations can then be performed on the entire system, permitting each molecule to relax according to the bond, angle and dihedral constraints outlined in the widely-used OPLS-AA forcefield, while still constraining the moieties to the coarse-grained centers-of-mass[51]. As a first pass, the non-bonded pair interaction potentials are initially disabled, and only the intra-molecular constraints affect the conformation of the molecules, effectively relaxing each in isolation. This first molecular dynamics phase has the result of straightening out the molecules, flipping the incorrectly oriented thiophene rings round to an energetically favorable rotation, and permitting the alkyl side-chains to flex and bend realistically based on the two coarse-grained sites (Figure 8b). However, with no repulsive potentials activated between non-bonded pairs, a significant number of atoms within the morphology overlap with those belonging to neighboring molecules.
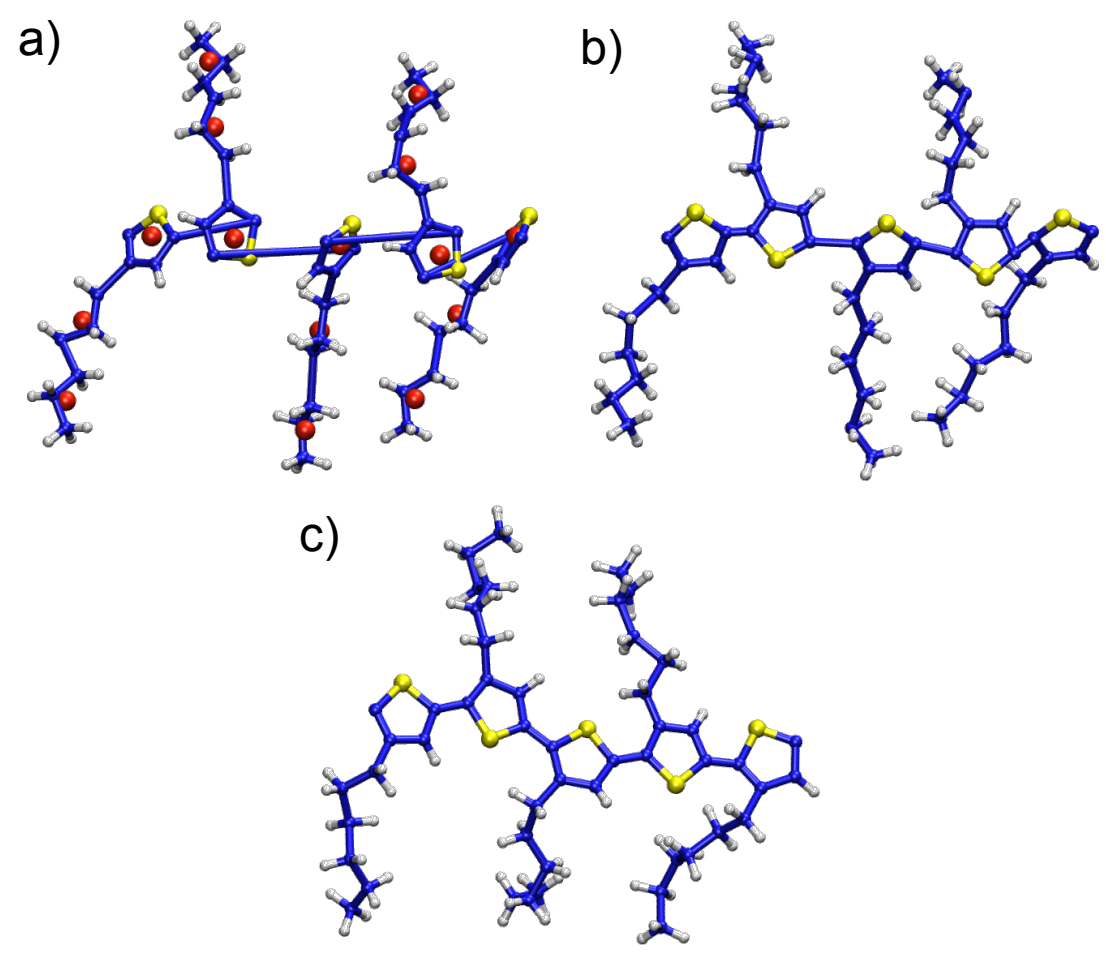

Figure 8. A representative example molecule of P3HT after the various molecular dynamics simulation phases discussed in the text for the fine-graining process: a) the initial projection of the molecule, b) the molecule relaxed according to the intra-chain potentials, c) the final, atomistic conformation of the molecule after considering pairwise potentials arising from nearby molecules. Blue atoms are carbon, white are hydrogen and yellow atoms represent sulfur. Red beads represent the centers-of-mass from the coarse-grained simulations.

Activating a hard non-bonded pair interaction, such as the commonly used Lennard-Jones (LJ) potential, can often lead to extremely large repulsive forces in situations where atoms overlap significantly. These forces cause numerical instabilities, strongly disrupting the molecule's equilibrium 
formation determined by the coarse-grained molecular dynamics. It is therefore not uncommon to use a "soft", repulsive pair potential such as the dissipative particle dynamics (DPD) forcefield, which is finite and well-defined at short separations, to gradually spread apart atoms, while still permitting them to overlap[72]. Some flavors of this potential include random fluctuations to the inter-atomic force, providing thermal 'kicks' or damping[87], however in this investigation, the DPD forcefield is not being used to obtain the final molecular conformations and is instead used as a tool to reduce overlap, so these fluctuations are unnecessary. After the overlap has been sufficiently reduced throughout the morphology, a harder LJ potential can be included in the molecular dynamics simulations to locate atoms correctly in the potential well that describes the balance between atomic repulsion and van der Waals attraction.

The example molecule's final conformation is shown in Figure 8c. This fine-graining methodology results in a morphology with complete molecules (sidechains included) in a geometrically optimized conformation with respect to themselves and their neighbors, while maintaining the center-ofmass positions determined by the coarse-grained molecular dynamics relaxation. Furthermore, this procedure is fully transferable to any other molecule with defined OPLS-AA forcefield parameters, providing that the coarse-graining scheme is fully described.

\section{Charge Mobility}

When an atomistic morphology has been obtained, the carrier mobility, $\mu$, within the system can be determined, which is a measure of how quickly free charges can move through the active layer. Charge transport within organic electronic devices proceeds through a series of quantum tunneling events, occurring between many 'hopping sites' throughout the morphology. The initial and destination hopping sites ( $i$ and $j$ respectively) are the frontier molecular orbitals of nearby chromophores - the highest occupied molecular orbital (HOMO) for holes and the lowest unoccupied molecular orbital (LUMO) for electrons. Each chromophore is a region over which a hole's (electron's) wavefunction at the HOMO (LUMO) energy is assumed to be fully delocalized. The extent and spatial locations of chromophores can be determined using QCCs. For some materials, such as polycyclic aromatic hydrocarbons, the molecules are small enough that a carrier's wavefunction has non-negligible probability density across the entirety of the molecule[88]. In larger, or more complex semiconductors, a single molecule may well have several strongly localized frontier molecular orbitals[89]. In the example case of P3HT, electronic structure calculations have predicted that each chromophore corresponds to a region spanning approximately 7 monomers along the chain backbone[90, 91].

The rate at which hopping can occur between chromophores, $k_{i \rightarrow j}$ is often given by the semiclassical Marcus expression[92]:

$$
k_{i \rightarrow j}=\frac{\left|T_{i j}\right|^{2}}{\hbar} \sqrt{\frac{\pi}{\lambda_{i j} k_{B} T}} \exp \left[-\frac{\left(\Delta E_{i j}-\lambda_{i j}\right)^{2}}{4 \lambda_{i j} k_{B} T}\right]
$$

where the prefactor depends on the electronic transfer integral between the initial and final chromophores, $T_{i j}$, and the reorganization energy, $\lambda_{i j}$. The exponential term contains the free-energy difference between the initial and final hopping states, $\Delta E_{i j}=E_{j}-E_{i}$, and therefore represents a penalty associated with hops across energies with magnitudes significantly greater than $\lambda_{i j}$. The majority of other investigations that employ this semi-classical Marcus treatment use a lattice-based model of a complete active layer, the morphology of which can be inferred from MD simulations[93], or by numerical methods such as modified Cahn-Hilliard[94-97] or Ising model techniques[98-100]. In these cases, the free energy, $\Delta E_{i j}$, takes into account any local variation in the energy levels (which is often selected from a density of state (DoS) distribution), the Coulomb interactions between nearby charge carriers and their images, as well as any contributions from the applied 
electric field across the morphology[96, 97, 101, 102]. For the current investigation, we consider the zero-field, time-of-flight hole mobility (which characteristically occurs at low charge densities) in the bulk with no electrical contacts (and therefore no image charges). The sole contributor to $\Delta E_{i j}$ is therefore the density of available states for the charge carriers. Previous studies have shown reasonable agreement between the distribution of HOMO energies for each chromophore and the experimentally observed DoS, and so for this investigation, $\Delta E_{i j}$ is taken to be the difference in HOMO levels between chromophores $i$ and $j[80]$.

$T_{i j}$ is a measure of the orbital overlap between chromophores. If the initial and destination sites are spatially proximal, then there is increased overlap between the frontier molecular orbitals and it becomes easier for charge carriers to hop between them, giving a faster rate, $\mathrm{k}_{i \rightarrow j}$. Generally, transfer integrals are approximated by using the energy splitting in dimer method[74, 103, 104], based on the framework of the Marcus-Hush two-state model[105, 106]. For the charge transport of holes, this operates on the assumption that, as a pair of chromophores are brought closer together from isolation, the HOMO level of the dimer splits, producing new HOMO and HOMO-1 energy levels. The magnitude of that splitting, when compared to the HOMO levels of each chromophore in isolation, can be directly related to the transfer integral by:

$$
\left|T_{i j}\right|=\frac{1}{2} \sqrt{\left(E_{\mathrm{HOMO}}-E_{\mathrm{HOMO}-1}\right)^{2}-\left(\Delta E_{i j}\right)^{2}}
$$

where $\left(E_{\mathrm{HOMO}}-E_{\mathrm{HOMO}-1}\right)$ denotes the HOMO splitting energy. It is important to note that many studies predict a more broad density-of-states for the chromophores than is expected experimentally[80, 107-110]. Therefore, some studies have successfully utilized Koopman's approximation, which effectively maps the density-of-states to a single narrow peak by assuming that each chromophore is identical with $\Delta E_{i j}=0[104,111,112]$. In order to map the transfer integrals for an entire morphology, which may contain many tens of thousands of chromophore pairs, a high throughput technique is required to determine the molecular orbital energies with high computational efficiency. The semi-empirical, ZINDO/S (Zerner's intermediate neglect of differential overlap) method can be used to quickly determine the required orbital energies, taking of the order seconds for each chromophore pair[113, 114]. These and similar calculations have shown to provide order-of-magnitude agreement with more rigorous density functional theory techniques[115, 116]. Due to the large number of independent calculations to be performed for each morphology, it is useful to leverage high performance computing clusters here, splitting the ZINDO/S calculations to run simultaneously over several cores, increasing the throughput.

The reorganization energy, $\lambda_{i j}$, describes the energy required to polarize and depolarize a chromophore as a charge carrier hops onto and subsequently off a particular site. It consists of two contributions, the 'internal' $\left(\lambda_{\text {int }}\right)$ and 'external' $\left(\lambda_{\text {ext }}\right)$ energies, which correspond to the normal frequency modes of the molecule and the contribution due to the reorientation of nearby molecules responding to the presence of the charge respectively[117]. While both contributions are material dependent, density functional theory and alternative theoretical treatments predict that $\lambda_{\text {ext }}$ is independent of chromophore length, whereas $\lambda_{\text {int }}$ decreases monotonically for longer chromophores[117-119]. Some investigations have had success from approximating the reorganization energy to be twice the charge carrier polaron energy, which can be tuned to match the simulated material[100, 120, 121], rather than explicitly calculating $\lambda_{i j}$ through QCCs or molecular dynamics[122, 123].

The Marcus theory used in this investigation is known to have a specific domain of validity, based on the magnitudes of $\Delta E_{i j}, T_{i j}$ and $\lambda_{i j}$. While the complete justification of the application of Marcus theory is beyond the scope of the techniques and results of this section, we provide a discussion of the validity of our implementation of the theory in the supplementary information (SI Section $\S 2$ ).

An event-driven treatment is required to convert the carrier hopping rate in Equation 16 into 
mobility data for a given morphology. Arguably, some of the most successful and ubiquitous eventdriven techniques are Kinetic Monte Carlo (KMC) simulations, which employ a classical representation of charge within organic electronic devices. Carriers and excitons are represented as physical particles, each localized to a particular chromophore or lattice site. The various permitted behaviors of each particle can be determined by a series of rate coefficients, $k$, for the physical, kinetic processes that occur within the device. These are not just limited to carrier hopping - for example, many KMC studies have also considered photo and electrical injection, exciton transport and dissociation, and carrier recombination in order to simulate a complete electronic device[95, 97, 98, 100]. Generally, the simulations proceed as follows. The 'wait time' for each event to occur, $\tau$, is calculated using the Monte Carlo algorithm:

$$
\tau=-\frac{\ln x}{k}
$$

where $0 \leq x<1$ is a uniformly distributed random number. A queue is formed in ascending $\tau$ for each event that has been considered, with the first event in the queue representing the behavior that will occur next chronologically. The code then steps through the queue, executing the first event and increasing the total simulation time by $\tau$. All subsequent event times in the queue are then decreased by $\tau$. The particle which has just been acted upon then has its new behavior calculated. If the executed event produced any new particles (as is the case for photo or electrical injection events) then the possible events for these particles are also calculated and then inserted into the queue in the appropriate, chronological position. The algorithm repeats until certain simulation termination criteria have been met, such as a carrier hopping for a particular distance[80] or when the flux of charge carriers hopping out of the active layer through the electrical contacts has converged to a particular value, after a certain number of photoinjections have taken place within the device[95, 121].

There are two main KMC methodologies, here termed 'mesoscale' and 'molecular'. In mesoscale KMC, the simulation volume consists of a regular, 3D cubic lattice, with dimensions comparable to the thickness of the thin-film active layer $(\sim 100 \mathrm{~nm})$, split into sites with side $\sim 1 \mathrm{~nm}$. These simulations are therefore capable of obtaining device characteristics for a given input morphology, which is usually generated using Cahn-Hilliard[95-97] or Ising $[98,124]$ theory. The ability to obtain device-scale characteristics, such as the power conversion efficiency or the $J$ - $V$ curve, comes at the price of abstracting out much of molecular interaction behavior that occurs on lengthscales $<1 \mathrm{~nm}$. Instead, the disordered energetic landscape resulting from these sub-lattice interactions is estimated using alternative methods[125, 126]. Commonly, a Gaussian Disorder Model is used, which applies a perturbation to a site's energy, selected randomly from a Gaussian distribution with a typical standard deviation of $\sigma=100 \mathrm{meV}[127,128]$, inspired by explicit QCCs of charge carrier densities within the material[129, 130]. This is, therefore, an approximate technique that implicitly assumes some degree of averaging of the electronic couplings in the morphology, while still going some way to consider the effects of molecular conformation on the bulk device performance.

Molecular KMC simulations attempt to explicitly consider these molecular effects on the charge and energy transport mechanisms of a device. To accomplish this, they operate at a higher resolution than their mesoscale counterparts, on the Angström lengthscale, therefore simulating regions of a morphology of side up to $\sim 10 \mathrm{~nm}[80]$. This makes molecular KMC less suited to exploring full device performance, but rather obtaining charge transport characteristics, such as the carrier mobility, within the representative bulk or at pre-constructed interfaces between materials.

The charge carrier mobility can be obtained for a molecular morphology by calculating $k_{i \rightarrow j}$ for every pair of chromophores within the system, according to Equation 16, as outlined above. A charge carrier can then be injected onto a random chromophore within the morphology. The Monte Carlo algorithm (Equation 18) can then be used to determine the timescales with which each possible hop from the initial chromophore can take place. The event with the shortest hop-time 
can then be selected as the most probable hop for the carrier, and the carrier can be moved to its destination, increasing the simulation time by $\tau$. Any hop that takes the carrier over the periodic boundary wraps it back into the system on the opposite side, in an attempt to account for the small simulation volumes compared to the active layer thickness. After many hops, the carrier will have moved through the system for a total time, $t$. The mean squared displacement for the carrier $\left(\left\langle x^{2}\right\rangle\right)$ can be calculated, the results averaged over $\sim 10,000$ carriers, and repeated for several values of $t$. There is an increasing, linear trend between $\left\langle x^{2}\right\rangle$ and $t$, the gradient of which corresponds to the $3 \mathrm{D}$ diffusion coefficient, $D$. One can relate $D$ to the zero-field mobility $\mu_{0}$ by using the Einstein relation:

$$
\mu_{0}=\frac{q D}{6 k_{B} T}
$$

where $k_{B}$ is the Boltzmann constant and $T$ is the temperature of the system. Here, the factor of $1 / 6$ is to account for dimensionality in the calculation, due to diffusive motion in 6 directions[131]. It is important to note that, while the carriers are hopping, no molecular motion is considered and the atoms within the morphology are frozen, instrinsically assuming that structural decorrelation occurs on timescales significantly longer than carrier propagation, and therefore any fluctuations in the local energetic structure of the morphology are negligible for the duration of the KMC simulations. A discussion of the appropriate timescales and the justification of this assumption is given in the supplementary information (SI Section $§ 2.1$ ). This mobility calculation is more similar to time-of-flight experiments, where low excitation fluence, charge density, and active layer thickness are required[132, 133], than field-effect transistor measurements on complete devices, which tend to report mobilities several orders of magnitude greater[134-136].
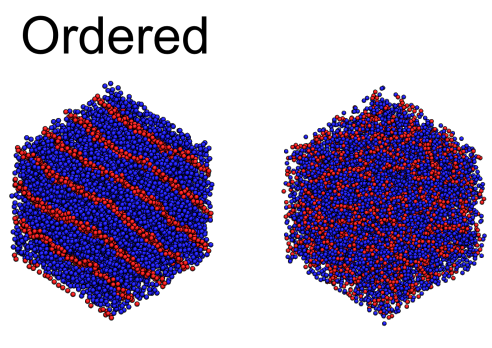

Disordered
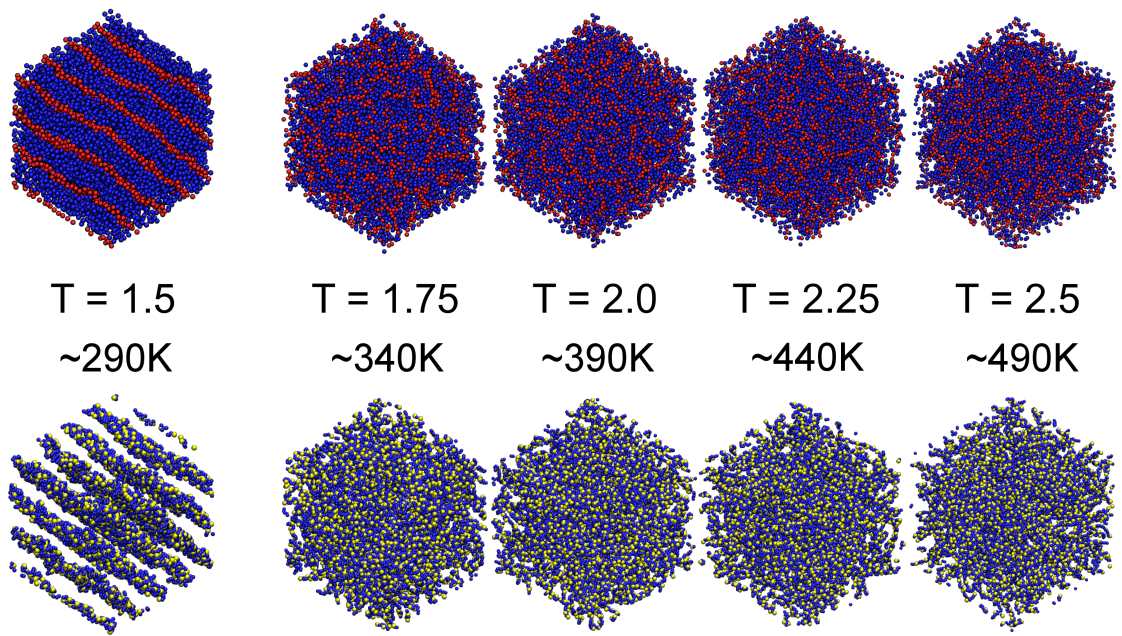

Figure 9. (top) The coarse-grained P3HT morphologies used in this investigation to emulate the bulk structure of the organic thin-film from Ref. [39]. Red beads depict the polymer backbone (site $P 1$ in Figure 7 a), and the blue elements depict sidechains (sites P2 and P3). The simulation temperatures are also shown for each system, along with each corresponding atomistic representation after the fine-graining process outlined above (bottom, with sidechain atoms omitted for clarity). In the atomistic morphologies, blue and yellow atoms represent the carbons and sulfur of each thiophene ring respectively.

To test the charge mobility calculation pipleline outlined above, we use a selection of coarsegrained P3HT morphologies that are expected to be representative of the bulk structure of a pristine thin film. The morphologies are depicted in Figure 9, where each simulation volume is a cube of side $\sim 2 \mathrm{~nm}$, containing 250 oligomers of length 15 monomers. Each system has been annealed at $\sim 490 \mathrm{~K}$, before being cooled by a series of consecutive quenches to a final simulation 
temperature, $T$, as described in Jankowski, Marsh, and Jayaraman[39]. The morphology exhibits an order-disorder transition at a critical temperature, $290 \mathrm{~K} \leq T_{c}<340 \mathrm{~K}$, and so there is one ordered morphology where the chains have $\pi$-stacked into lamellae, and four amorphous systems with little to no long-range order. The atomistic representations shown in Figure 9 have been obtained using the robust, molecular dynamics-based fine-graining methodology outlined in the previous section. For the charge transport calculation, each chromophore consisted of a single monomer, rather than the average carrier delocalization of 7 monomers as determined experimentally[90, 91]. Previous investigations of this type have found it challenging to determine a computationally efficient methodology to calculate chromophore locations along a contorted chain that retains good quantitative agreement with carrier mobilities[80]. This, coupled with the short length of the simulated chains, suggests that a more basic chromophore identification regime would be better suited to this investigation. As such, the mobility calculations are performed on chromophores of length 15 monomers (the entirety of each molecule, effectively assuming instantaneous intra-chain transport) and chromophores of length 1 monomer. While intra-chain transport is assumed to be significantly faster than inter-chain hopping, the assumption that transport along the chain backbone is instantaneous for these molecules is found to result in unrealistically high mobilities. Indeed, first-principles investigations of Gaussian and worm-like chains have suggested that intrachain hopping occurs on timescales only one or two orders of magnitude faster than inter-chain transport[131]. Chromophores consisting of a single monomer however, were found to well replicate the ratio between inter- and intra-chain transport, resulting in realistic mobilities for the systems. As such, we proceed considering only the single-monomer chromophore case. Carrier hopping rates between monomers are calculated using Equation 16, with $\lambda_{i j}=300 \mathrm{meV}[118]$.
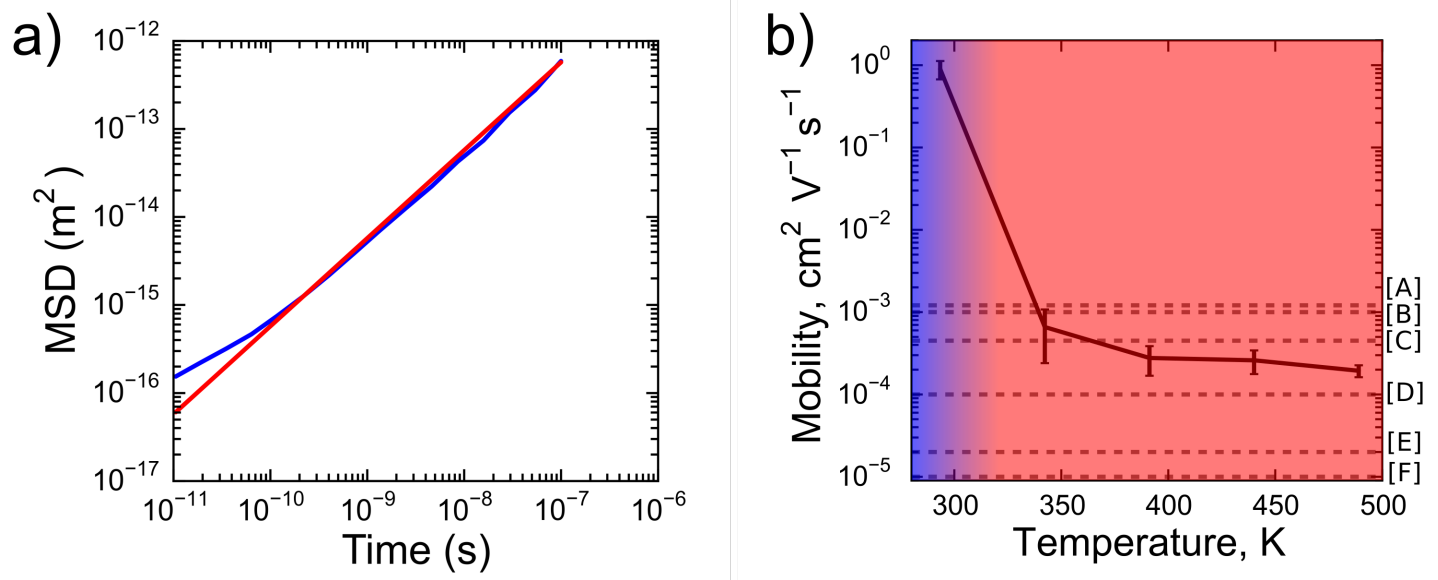

Figure 10. Charge mobility results for the test P3HT morphologies studied in this investigation. a) An example of the linear trend between mean squared displacement and time. The blue line describes the data obtained from the Kinetic Monte Carlo simulations, and the red line shows the line of best fit. b) The calculated hole mobilities of the pristine P3HT thin films explored (solid lines). Error bars were calculated from multiple runs of morphologies generated with the same statepoint, but statistically independent structures. The red and blue regions denote disordered and ordered regimes respectively. The dashed lines show example experimental mobilities for comparable systems from literature references: [A][137], [B][138], [C][110], [D][108, 110], $[\mathrm{E}][110],[\mathrm{F}][108,110,138]$

An example mean squared displacement trend is shown in Figure 10a, for the ordered morphology, with an $\mathrm{r}^{2}$ correlation coefficient of 0.995 in calculating the diffusion coefficient. The calculated zero-field hole mobilities for each of the investigated systems are shown in Figure 10b. The expected qualitative trend in mobility has been replicated; the mobility of the ordered morphology is significantly greater than the mobility within the disordered morphologies. Furthermore, the pipeline has quantitatively predicted that the improvement in mobility is around three orders of magnitude, in reasonable agreement with experimental investigations which have demonstrated a 1-2 order of 
magnitude increase in time-of-flight and field-effect mobilities depending on processing conditions, crystallinity and morphological structure[139-142]. Additionally, the absolute values of the mobilities reported here are in good quantitative agreement with experimental results. In the amorphous and semi-crystalline phases, P3HT tends to exhibit a time-of-flight hole mobility of between $1 \times 10^{-5}$ $-1 \times 10^{-3} \mathrm{~cm}^{2} \mathrm{~V}^{-1} \mathrm{~s}^{-1}$ depending on the processing conditions[108, 110, 137, 138]. This is in excellent agreement with the disordered mobilities obtained in Figure 10b, for which $1 \times 10^{-4} \leq \mu_{0}<$ $3 \times 10^{-4} \mathrm{~cm}^{2} \mathrm{~V}^{-1} \mathrm{~s}^{-1}$. For the ordered morphology, $\mu_{0} \sim 0.1 \mathrm{~cm}^{2} \mathrm{~V}^{-1} \mathrm{~s}^{-1}$ may seem unrealistically high for time-of-flight mobility measurements at low charge densities, but it matches well the expected field-effect transistor measurements obtained experimentally[143, 144]. We emphasize that the near-perfectly ordered P3HT morphology studied here lacks the grain boundaries and defects present in experimental samples of P3HT assembled by chains with hundreds or thousands of monomers. It stands to reason that these simulated oligomer morphologies should have enhanced carrier mobility relative to experimental observations of polycrystalline and paracrystalline films.

In addition to the new results from this investigation, similar combinations of simulation techniques to those outlined here have been used successfully in the literature to faithfully recreate trends observed experimentally. For instance, many literature investigations have successfully studied the effect of modifying the relative size, position and orientation of chromophores on variations in the electronic structure of organic semiconducting materials, including the density of states[107, 108, 110], transfer integrals[74, 104, 145] and reorganization energies[122, 123, 146]. Although several studies report excellent agreement with other literature sources for many of the components required to calculate the charge transport characteristics of a particular system, it is common for investigations to omit reporting a final mobility value[104, 147, 148]. A possible reason for this could be that, while qualitative agreement with experiment is common, quantitative agreement can vary significantly depending on the system studied. In general, calculated mobility values are often more accurate in small molecule systems where transfer integrals are averaged over, or an initial configuration is assumed[141, 149, 150]. Otherwise, these computational methods tend to overpredict the time-of-flight mobilities of material systems by two to six orders of magnitude, with more noticeable discrepancies for larger or more complex molecules with a greater quantity of physical conformations, such as polymer chains[75, 80, 151, 152].

The fine-graining and charge-transport calculations outlined in this article attempt to address some of the issues associated with linking molecular morphology to charge transport. Specifically, for complex molecules such as oligomers or polymer chains, employing molecular dynamics simulations allows realistic conformations to be obtained within a large, coarse-grained, pre-relaxed morphology. Additionally, removing assumptions about the differences between inter- and intra-chain transport and carrier delocalization by instead using QCCs to determine the electronic couplings between small chromophores in-situ, the quantitative discrepancy between these investigations and experimental data appears to be reduced. Coupling charge transport studies with structural analysis of the electrical network[122, 153, 154] or crystal packing[80, 123], can help predict the morphological features that provide the best device performance. Extending the scope of the KMC simulations beyond the bulk, pristine domain to molecular and electrical interfaces for different materials allows for the prediction of full device performance characteristics such as power conversion efficiencies and $J-V$ curves. With these data, a set of design rules may be obtained, which can suggest the best molecules and processing conditions to optimize the efficiency of many organic electronic devices.

\section{Conclusions}

Using a combination of coarse-grained models, GPU-accelerated molecular dynamics, careful finegraining, and quantum chemical calculations it is now possible to infer the charge mobility characteristics of OPV active layer morphologies generated from computer experiments. These capabilities 
are the first step towards determining which morphologies have the best charge transport properties for a given chemistry and the factors that govern high charge mobility. Further, this framework will enable the high-throughput screening of active layer ingredients for those with highest self-assembly propensity for efficient OPV morphologies.

Additional advances are needed to complement and extend this work to realize the goal of predicting which ingredients and manufacturing protocols will produce the highest-efficiency OPVs. Modeling the temperature gradients, solvent evaporation, and fluid flows in roll-to-roll manufacturing processes will require sophisticated add-ons to existing MD software or new nonequilibrium simulation techniques. There are also opportunities to study morphology at the anode and cathode interfaces of the active layer, where details of morphology are harder to probe and the transport of charges is especially important to overall power conversion efficiency. Which electrode materials have the best electronic properties for each combination of active layer ingredients? How do those surfaces influence the morphology of active layer ingredients nearby? Answering these questions will depend on the development of validated interaction potentials describing organic and inorganic force fields and access to even longer simulatoin timescales. These opportunities to contribute to the fundamental understanding and engineering of OPVs will not only advance the ability to engineer low-cost sustainable power sources, but also improve the predictive capabilities of molecular simulations used to study biomolecular systems, nanoparticle self-assembly, complex fluids, and nonequilibrium systems of energetically-driven particles.

\section{Acknowledgements}

This work used the Extreme Science and Engineering Discovery Environment (XSEDE), which is supported by National Science Foundation grant number ACI-1053575[155]. This material is based upon work supported by the National Science Foundation under Grant No. (1229709).

\section{References}

[1] Nieves Espinosa, Markus Hösel, Dechan Angmo, and Frederik C. Krebs. Solar cells with one-day energy payback for the factories of the future. Energy Environ. Sci., 5:5117, 2012.

[2] Katherine A. Mazzio and Christine K Luscombe. The future of organic photovoltaics. Chem. Soc. Rev., 44(1):78-90, sep 2015.

[3] M Kaushik and Bharat Kaushik. Organic Solar Cells: Design, Synthesis and Characterization. Int. J. Eng. Sci., 2(July):310-319, 2013.

[4] Jeffrey Peet, Alan J Heeger, and Guillermo C Bazan. "Plastic" solar cells: self-assembly of bulk heterojunction nanomaterials by spontaneous phase separation. Acc. Chem. Res., 42(11):1700-1708, nov 2009.

[5] Ryan C Chiechi, Remco W.a. Havenith, Jan C Hummelen, L Jan Anton Koster, and Maria a. Loi. Modern plastic solar cells: materials, mechanisms and modeling. Mater. Today, 16(7-8):281-289, jul 2013.

[6] Koen Vandewal, Scott Himmelberger, and Alberto Salleo. Structural factors that affect the performance of organic bulk heterojunction solar cells. Macromolecules, 46:6379-6387, 2013.

[7] Kenneth R Graham, Clement Cabanetos, Justin P Jahnke, Matthew N Idso, Abdulrahman El Labban, Guy O. Ngongang Ndjawa, Thomas Heumueller, Koen Vandewal, Alberto Salleo, Bradley F Chmelka, Aram Amassian, Pierre M Beaujuge, and Michael D. McGehee. Importance of the Donor:Fullerene Intermolecular Arrangement for High-Efficiency Organic Photovoltaics. J. Am. Chem. Soc., 136(27):9608-9618, jul 2014.

[8] Sharon C Glotzer. Assembly engineering: Materials design for the 21st century (2013 P.V. Danckwerts lecture). Chem. Eng. Sci., oct 2014.

[9] P. Hohenberg and W. Kohn. Inhomogeneous Electron Gas. Phys. Rev., 136(3B):B864-B871, nov 1964. 
[10] A. Szabo and N. S. Ostlund. Modern Quantum Chemistry: Introduction to Advanced Electronic Structure Theory. Courier Corporation, 1989.

[11] E. K. U. Gross, J. F. Dobson, and M. Petersilka. Density Functional Theory of Time-Dependent Phenomena. In Density Funct. Theory II, pages 81-172. Springer-Verlag, Berlin/Heidelberg, 1996.

[12] Sharon E Koh, Bernard Delley, Julia E Medvedeva, Antonio Facchetti, Arthur J Freeman, Tobin J Marks, and Mark A Ratner. Quantum Chemical Analysis of Electronic Structure and n- and p-Type Charge Transport in Perfluoroarene-Modified Oligothiophene Semiconductors. J. Phys. Chem. B, 110(48):24361-24370, dec 2006.

[13] R. Schueppel, K. Schmidt, C. Uhrich, K. Schulze, D. Wynands, J. L. Brédas, E. Brier, E. Reinold, H.-B. Bu, P. Baeuerle, B. Maennig, M. Pfeiffer, and K. Leo. Optimizing Organic Photovoltaics using Tailored Heterojunctions: A Photoinduced Absorption Study of Oligothiophenes with Low Band Gaps. Phys. Rev. B, 77(8):085311, feb 2008.

[14] T. Helgaker, P. Jorgensen, and J. Olsen. Molecular Electronic-Structure Theory. John Wiley \& Sons Ltd., 2014.

[15] Christian L Uhrich, Christiane Falkenberg, Jan Rabe, Bruno Heimke, Matthias Klemet, Christian Wilde, Ralph Wichtendahl, and Martin Pfeiffer. Organic solar cells: from lab to roll-to-roll production. 9184:918415, oct 2014 .

[16] Xiaohui Lin, Harish Subbaraman, Zeyu Pan, Amir Hosseini, Chris Longe, Klay Kubena, Paul Schleicher, Phillip Foster, Sean Brickey, and Ray Chen. Towards Realizing High-Throughput, Roll-to-Roll Manufacturing of Flexible Electronic Systems. Electronics, 3(4):624-635, 2014.

[17] Nichole Cates Miller, Sean Sweetnam, Eric T Hoke, Roman Gysel, Chad E Miller, Jonathan a Bartelt, Xinxin Xie, Michael F Toney, and Michael D McGehee. Molecular Packing and Solar Cell Performance in Blends of Polymers with a Bisadduct Fullerene. Nano Lett., 12(3):1566-1570, mar 2012.

[18] Wei Chen, Tao Xu, Feng He, Wei Wang, Cheng Wang, Joseph Strzalka, Yun Liu, Jianguo Wen, Dean J Miller, Jihua Chen, Kunlun Hong, Luping Yu, and Seth B Darling. Hierarchical nanomorphologies promote exciton dissociation in polymer/fullerene bulk heterojunction solar cells. Nano Lett., 11(9):3707-3713, sep 2011.

[19] Shuyan Shao, Jian Liu, Jidong Zhang, Baohua Zhang, Zhiyuan Xie, Yanhou Geng, and Lixiang Wang. Interface-induced crystalline ordering and favorable morphology for efficient annealing-free poly(3hexylthiophene): Fullerene derivative solar cells. ACS Appl. Mater. Interfaces, 4:5704-5710, 2012.

[20] Eric Verploegen, Rajib Mondal, Christopher J Bettinger, Seihout Sok, Michael F Toney, and Zhenan Bao. Effects of Thermal Annealing Upon the Morphology of Polymer-Fullerene Blends. Adv. Funct. Mater., 20(20):3519-3529, oct 2010.

[21] Stefan Grob, Andrew N. Bartynski, Andreas Opitz, Mark Gruber, Florian Grassl, Eduard Meister, Theresa Linderl, Ulrich Hörmann, Christopher Lorch, Ellen Moons, Frank Schreiber, Mark E. Thompson, and Wolfgang Brütting. Solvent vapor annealing on perylene-based organic solar cells. J. Mater. Chem. A, 3(30):15700-15709, 2015.

[22] Valerie Daggett. Molecular Dynamics Simulations of the Protein Unfolding/Folding Reaction. Acc. Chem. Res., 35(6):422-429, jun 2002.

[23] David N LeBard, Benjamin G Levine, Philipp Mertmann, Stephen A Barr, Arben Jusufi, Samantha Sanders, Michael L Klein, Athanassios Z Panagiotopoulos, A Barr, Arben Jusufi, Samantha Sanders, and L Klein. Self-assembly of coarse-grained ionic surfactants accelerated by graphics processing units. Soft Matter, 8(8):1-5, 2011.

[24] Robert B Best, Gerhard Hummer, and William A Eaton. Native contacts determine protein folding mechanisms in atomistic simulations. Proc. Natl. Acad. Sci. U. S. A., 110(44):17874-17879, oct 2013.

[25] B Buesser, A. J. Grohn, and S E Pratsinis. Sintering Rate and Mechanism of TiO 2 Nanoparticles by Molecular Dynamics. J. Phys. Chem. C, 115(22):11030-11035, jun 2011.

[26] Robert M. Elder and Arthi Jayaraman. Structure and thermodynamics of ssDNA oligomers near hydrophobic and hydrophilic surfaces. Soft Matter, 9(48):11521, 2013.

[27] Amir Haji-Akbari, Michael Engel, Aaron S Keys, Xiaoyu Zheng, Rolfe G Petschek, Peter PalffyMuhoray, and Sharon C Glotzer. Disordered, quasicrystalline and crystalline phases of densely packed tetrahedra. Nature, 462(7274):773-777, dec 2009.

[28] Amir Haji-Akbari, Michael Engel, and Sharon C Glotzer. Phase Diagram of Hard Tetrahedra. J. Chem. Phys., 135(19):194101:1-194101:10, nov 2011.

[29] Joshua A Anderson, Chris D Lorenz, and A Travesset. General Purpose Molecular Dynamics Simu- 
lations Fully Implemented on Graphics Processing Units. J. Comput. Phys., 227(10):5342-5359, may 2008.

[30] Christian Robert Trott. LammpsCuda-a new GPU accelerated Molecular Dynamics Simulations Package and its Application to Ion-Conducting Glasses. PhD thesis, 2011.

[31] John E Stone, James C Phillips, Peter L Freddolino, David J Hardy, Leonardo G Trabuco, and Klaus Schulten. Accelerating molecular modeling applications with graphics processors. J. Comput. Chem., 28(16):2618-2640, dec 2007.

[32] Benjamin G Levine, John E Stone, and Axel Kohlmeyer. Fast Analysis of Molecular Dynamics Trajectories with Graphics Processing Units-Radial Distribution Function Histogramming. J. Comput. Phys., 230(9):3556-3569, may 2011.

[33] Joshua A Anderson and Sharon C Glotzer. The development and expansion of HOOMD-blue through six years of GPU proliferation. arXiv, 1308.5587, aug 2013.

[34] Joshua L Phillips, Michael E Colvin, and Shawn Newsam. Validating clustering of molecular dynamics simulations using polymer models. BMC Bioinformatics, 12:445, 2011.

[35] Joshua A. Anderson, Eric Jankowski, Thomas L. Grubb, Michael Engel, and Sharon C. Glotzer. Massively parallel monte carlo for many-particle simulations on GPUs. J. Comput. Phys., 254:27-38, dec 2013.

[36] Jens Glaser, Jian Qin, Pavani Medapuram, and David C Morse. Collective and Single-Chain Correlations in Disordered Melts of Symmetric Diblock Copolymers: Quantitative Comparison of Simulations and Theory. Macromolecules, 47(2):851-869, jan 2014.

[37] Andreas W. Götz, Mark J. Williamson, Dong Xu, Duncan Poole, Scott Le Grand, and Ross C Walker. Routine Microsecond Molecular Dynamics Simulations with AMBER on GPUs. 1. Generalized Born. J. Chem. Theory Comput., 8(5):1542-1555, may 2012.

[38] Jan-Michael Y Carrillo, Rajeev Kumar, Monojoy Goswami, Bobby G Sumpter, and W Michael Brown. New insights into the dynamics and morphology of P3HT:PCBM active layers in bulk heterojunctions. Phys. Chem. Chem. Phys., 15(41):17873, nov 2013.

[39] Eric Jankowski, Hilary S. Marsh, and Arthi Jayaraman. Computationally Linking Molecular Features of Conjugated Polymers and Fullerene Derivatives to Bulk Heterojunction Morphology. Macromolecules, 46(14):5775-5785, jul 2013.

[40] Hilary S Marsh, Eric Jankowski, and Arthi Jayaraman. Controlling the Morphology of Model Conjugated Thiophene Oligomers through Alkyl Side Chain Length, Placement, and Interactions. Macromolecules, 47(8):2736-2747, apr 2014.

[41] William G. Hoover. Canonical Dynamics: Equilibrium Phase-space Distributions. Phys. Rev. A, 31(3):1695-1697, 1985.

[42] M. Parrinello and A. Rahman. Polymorphic transitions in single crystals: A new molecular dynamics method. J. Appl. Phys., 52(12):7182-7190, 1981.

[43] Glenn J. Martyna, Douglas J. Tobias, and Michael L. Klein. Constant pressure molecular dynamics algorithms. J. Chem. Phys., 101(September):4177, 1994.

[44] Florian Muller-Plathe. A simple nonequilibrium molecular dynamics method for calculating the thermal conductivity. J. Chem. Phys., 106(14):6082, 1997.

[45] Tamio Ikeshoji and Bjørn Hafskjold. Non-equilibrium molecular dynamics calculation of heat conduction in liquid and through liquid-gas interface. Mol. Phys., 81(2):251-261, feb 1994.

[46] F Müller-Plathe. Reversing the perturbation in nonequilibrium molecular dynamics: An easy way to calculate the shear .... Phys. Rev. E, 59(5):4894-4898, 1999.

[47] Florian Müller-Plathe. Coarse-graining in polymer simulation: from the atomistic to the mesoscopic scale and back. Chemphyschem, 3(9):755-769, sep 2002.

[48] Helgi I. Ingólfsson, Cesar a. Lopez, Jaakko J Uusitalo, Djurre H de Jong, Srinivasa M Gopal, Xavier Periole, and Siewert J Marrink. The power of coarse graining in biomolecular simulations. Wiley Interdiscip. Rev. Comput. Mol. Sci., 4(3):225-248, may 2014.

[49] Siewert J. Marrink, Alex H de Vries, and Alan E. Mark. Coarse Grained Model for Semiquantitative Lipid Simulations. J. Phys. Chem. B, 108(2):750-760, jan 2004.

[50] Siewert J Marrink, H Jelger Risselada, Serge Yefimov, D Peter Tieleman, and Alex H De Vries. The MARTINI Force Field : Coarse Grained Model for Biomolecular Simulations The MARTINI Force Field : Coarse Grained Model for Biomolecular Simulations. J. Phys. Chem. B, 111(June):7812-7824, 2007. 
[51] William L Jorgensen and Julian. Tirado-Rives. The OPLS [Optimized Potentials for Liquid Simulations] Potential Functions for Proteins, Energy Minimizations for Crystals of Cyclic Peptides and Crambin. J. Am. Chem. Soc., 110(6):1657-1666, mar 1988.

[52] Timothy C Moore, Christopher R Iacovella, and Clare McCabe. Derivation of coarse-grained potentials via multistate iterative Boltzmann inversion. J. Chem. Phys., 140(22):224104, jun 2014.

[53] Timothy C. Moore, Christopher R. Iacovella, and Clare McCabe. Development of a coarse-grained water forcefield via multistate iterative Boltzmann inversion. arXiv, 1509.07887, sep 2015.

[54] Siewert J Marrink and D Peter Tieleman. Perspective on the Martini model. Chem. Soc. Rev., 42(16):6801-6822, aug 2013.

[55] Khanh Do, David M Huang, Roland Faller, and Adam J. Moulé. A comparative MD study of the local structure of polymer semiconductors P3HT and PBTTT. Phys. Chem. Chem. Phys., 12(44):14735, nov 2010.

[56] David M Huang, Roland Faller, Khanh Do, and Adam J. Moule. Coarse-Grained Computer Simulations of Polymer/Fullerene Bulk Heterojunctions for Organic Photovoltaic Applications. J. Chem. Theory Comput., 6(2):526-537, feb 2010.

[57] David M Huang, Adam J Moule, and Roland Faller. Characterization of PolymerFullerene Mixtures for Organic Photovoltaics by Systematically Coarse-Grained Molecular Simulations. Fluid Phase Equilib., 302(1-2):21-25, mar 2011.

[58] Sangwon Ko, Eric T Hoke, Laxman Pandey, Sanghyun Hong, Rajib Mondal, Chad Risko, Yuanping Yi, Rodrigo Noriega, Michael D McGehee, Jean-Luc Brédas, Alberto Salleo, and Zhenan Bao. Controlled conjugated backbone twisting for an increased open-circuit voltage while having a high short-circuit current in poly(hexylthiophene) derivatives. J. Am. Chem. Soc., 134(11):5222-5232, mar 2012.

[59] Victor Ho, Bryan W Boudouris, and Rachel a. Segalman. Tuning Polythiophene Crystallization through Systematic Side Chain Functionalization. Macromolecules, 43(19):7895-7899, oct 2010.

[60] Christopher Bruner, Nichole C. Miller, Michael D. McGehee, and Reinhold H. Dauskardt. Molecular intercalation and cohesion of organic bulk heterojunction photovoltaic devices. Adv. Funct. Mater., 23(22):2863-2871, 2013.

[61] Herbert Hauptman. The phase problem of x-ray crystallography, 1983.

[62] Dean M DeLongchamp, R Joseph Kline, and Andrew Herzing. Nanoscale Structure Measurements for Polymer-fullerene Photovoltaics. Energy Environ. Sci., 5(3):5980-5993, 2012.

[63] Klaus Schmidt-Rohr. Simulation of Small-angle Scattering Curves by Numerical Fourier Transformation. J. Appl. Crystallogr., 40:16-25, 2007.

[64] Sébastien Le Roux and Valeri Petkov. ISAACS-interactive structure analysis of amorphous and crystalline systems. J. Appl. Crystallogr., 43(1):181-185, 2010.

[65] D Frenkel. Simulations: The dark side. Eur. Phys. J. Plus, 128(1):10, jan 2013.

[66] Tsjerk A. Wassenaar, Kristyna Pluhackova, Rainer A. Böckmann, Siewert J. Marrink, and D. Peter Tieleman. Going Backward: A Flexible Geometric Approach to Reverse Transformation from Coarse Grained to Atomistic Models. J. Chem. Theory Comput., 10(2):676-690, feb 2014.

[67] Jerod Parsons, J. Bradley Holmes, J. Maurice Rojas, Jerry Tsai, and Charlie E M Strauss. Practical conversion from torsion space to cartesian space for in silico protein synthesis. J. Comput. Chem., 26(10):1063-1068, 2005.

[68] Matías R. Machado and Sergio Pantano. SIRAH tools: Mapping, backmapping and visualization of coarse-grained models. Bioinformatics, 32(10):1568-1570, 2016.

[69] V. A. Harmandaris, N. P. Adhikari, N. F. a. van der Vegt, and K. Kremer. Hierarchical Modeling of Polystyrene: From Atomistic to Coarse-Grained Simulations. Macromolecules, 39(19):6708-6719, sep 2006.

[70] Berk Hess, Salvador León, Nico van der Vegt, and Kurt Kremer. Long time atomistic polymer trajectories from coarse grained simulations: bisphenol-A polycarbonate. Soft Matter, 2(5):409-414, 2006.

[71] Giuseppe Santangelo, Andrea Di Matteo, Florian Müller-Plathe, and Giuseppe Milano. From mesoscale back to atomistic models: A fast reverse-mapping procedure for vinyl polymer chains. J. Phys. Chem. B, 111(11):2765-2773, 2007.

[72] Christine Peter, Luigi Delle Site, and Kurt Kremer. Classical simulations from the atomistic to the mesoscale and back: coarse graining an azobenzene liquid crystal. Soft Matter, 4(4):859, 2008.

[73] Xiaoyu Chen, Paola Carbone, Giuseppe Santangelo, Andrea Di Matteo, Giuseppe Milano, and Florian Müller-Plathe. Backmapping coarse-grained polymer models under sheared nonequilibrium conditions. 
Phys. Chem. Chem. Phys., 11(12):1977, 2009.

[74] Wei-Qiao Deng and William A. Goddard. Predictions of Hole Mobilities in Oligoacene Organic Semiconductors from Quantum Mechanical Calculations. J. Phys. Chem. B, 108(25):8614-8621, jun 2004.

[75] Jenny Nelson, Joe J Kwiatkowski, James Kirkpatrick, and Jarvist M Frost. Modeling Charge Transport in Organic Photovoltaic Materials. Acc. Chem. Res., 42(11):1768-1778, nov 2009.

[76] Kyra N Schwarz, Tak W Kee, and David M Huang. Coarse-Grained Simulations of the Solution-Phase Self-Assembly of poly(3-hexylthiophene) Nanostructures. Nanoscale, 5(5):2017-2027, mar 2013.

[77] Christine Peter and Kurt Kremer. Multiscale Simulation of Soft Matter Systems. Faraday Discuss., 144:9-24, 2010.

[78] Azadeh Ghanbari, Michael C. Bohm, and Florian Muller-Plathe. A Simple Reverse Mapping Procedure for Coarse-Grained Polymer Models with Rigid Side Groups. Macromolecules, 44(13):5520-5526, jul 2011.

[79] Guojie Zhang, Livia A. Moreira, Torsten Stuehn, Kostas Ch Daoulas, and Kurt Kremer. Equilibration of High Molecular Weight Polymer Melts: A Hierarchical Strategy. ACS Macro Lett., 3(2):198-203, feb 2014.

[80] M. L. Jones, D. M. Huang, B. Chakrabarti, and Chris Groves. Relating Molecular Morphology to Charge Mobility in Semicrystalline Conjugated Polymers. J. Phys. Chem. C, 120(8):4240-4250, 2016.

[81] Trung Dac Nguyen, Carolyn L Phillips, Joshua a. Anderson, and Sharon C Glotzer. Rigid Body Constraints Realized in Massively-parallel Molecular Dynamics on Graphics Processing Units. Comput. Phys. Commun., 182(11):2307-2313, nov 2011.

[82] Evan D. Miller, Matthew L. Jones, and Eric Jankowski. Enhanced computational sampling of perylene and perylothiophene packing with rigid-body models. ACS Omega,-Accepted, 2017.

[83] Hsiang-Yu Chen, Jianhui Hou, Shaoqing Zhang, Yongye Liang, Guanwen Yang, Yang Yang, Luping $\mathrm{Yu}$, Yue $\mathrm{Wu}$, and Gang Li. Polymer solar cells with enhanced open-circuit voltage and efficiency. Nat. Photonics, 3(11):649-653, nov 2009.

[84] Amit Babel and Samson A Jenekhe. Alkyl Chain Length Dependence of the Field-Effect Carrier Mobility in Regioregular poly(3-alkylthiophene)s. Synth. Met., 148(2):169-173, jan 2005.

[85] Seth B Darling and Michael Sternberg. Importance of Side Chains and Backbone Length in Defect Modeling of Poly(3-alkylthiophenes). J. Phys. Chem. B, 113(18):6215-6218, may 2009.

[86] Naga Rajesh Tummala, Chad Risko, Christopher Bruner, Reinhold H. Dauskardt, and Jean-Luc Brédas. Entanglements in P3HT and their Influence on Thin-Film Mechanical Properties: Insights from Molecular Dynamics Simulations. J. Polym. Sci. Part B Polym. Phys., 53(13):934-942, jul 2015.

[87] Robert D. Groot and Patrick B. Warren. Dissipative Particle Dynamics: Bridging the Gap between Atomistic and Mesoscopic Simulation. J. Chem. Phys., 107(11):4423, 1997.

[88] Sigma Hashimoto, Toshiaki Ikuta, Kazushi Shiren, Soichiro Nakatsuka, Jingping Ni, Masaharu Nakamura, and Takuji Hatakeyama. Triplet-Energy Control of Polycyclic Aromatic Hydrocarbons by BN Replacement: Development of Ambipolar Host Materials for Phosphorescent Organic Light-Emitting Diodes. Chem. Mater., 26(21):6265-6271, nov 2014.

[89] Aldilene Saraiva-Souza, Fabricio Macedo de Souza, Vicente F P Aleixo, Eduardo Costa Girao, Josue Mendes Filho, Vincent Meunier, Bobby G. Sumpter, Anto^nio Gomes Souza Filho, and Jordan Del Nero. A Single Molecule Rectifier with Strong Push-Pull Coupling. J. Chem. Phys., 129(20):204701, 2008.

[90] David P McMahon, David L Cheung, Ludwig Goris, Javier Dacuna, Alberto Salleo, and Alessandro Troisi. Relation between Microstructure and Charge Transport in Polymers of Different Regioregularity. J. Phys. Chem. C, 115(39):19386-19393, oct 2011.

[91] D H K Murthy, M Gao, M J W Vermeulen, L D A Siebbeles, and T J Savenije. Mechanism of Mobile Charge Carrier Generation in Blends of Conjugated Polymers and Fullerenes: Significance of Charge Delocalization and Excess Free Energy. J. Phys. Chem. C, 116(16):9214-9220, apr 2012.

[92] R A Marcus. Chemical and Electrochemical Electron-Transfer Theory. Annu. Rev. Phys. Chem., 15(1):155-196, oct 1964 .

[93] Naga Rajesh Tummala, Shafigh Mehraeen, Yao-Tsung Fu, Chad Risko, and Jean-Luc Brédas. Materials-Scale Implications of Solvent and Temperature on [6,6]-Phenyl-C61-butyric Acid Methyl Ester (PCBM): A Theoretical Perspective. Adv. Funct. Mater., 23(46):5800-5813, dec 2013.

[94] Ian C Henderson and Nigel Clarke. On Modelling Surface Directed Spinodal Decomposition. Macromol. Theory Simulations, 14(7):435-443, aug 2005. 
[95] B P Lyons, N Clarke, and C Groves. The Quantitative Effect of Surface Wetting Layers on the Performance of Organic Bulk Heterojunction Photovoltaic Devices. J. Phys. Chem. C, 115(45):2257222577, nov 2011.

[96] Benjamin P Lyons, Nigel Clarke, and Chris Groves. The Relative Importance of Domain Size, Domain Purity and Domain Interfaces to the Performance of Bulk-Heterojunction Organic Photovoltaics. Energy Environ. Sci., 5(6):7657, 2012.

[97] Matthew L. Jones, Reesha Dyer, Nigel Clarke, and Chris Groves. Are Hot Charge Transfer States the Primary Cause of Efficient Free-Charge Generation in Polymer:Fullerene Organic Photovoltaic Devices? A Kinetic Monte Carlo Study. Phys. Chem. Chem. Phys., 16(38):20310-20320, jun 2014.

[98] Peter K Watkins, Alison B Walker, and Geraldine L B Verschoor. Dynamical Monte Carlo Modelling of Organic Solar Cells: The Dependence of Internal Quantum Efficiency on Morphology. Nano Lett., 5(9):1814-1818, sep 2005.

[99] R. A. Marsh, C. Groves, and N. C. Greenham. A Microscopic Model for the Behavior of Nanostructured Organic Photovoltaic Devices. J. Appl. Phys., 101(8):083509, 2007.

[100] Chris Groves, Robin G E Kimber, and Alison B. Walker. Simulation of Loss Mechanisms in Organic Solar Cells: A Description of the Mesoscopic Monte Carlo Technique and an Evaluation of the First Reaction Method. J. Chem. Phys., 133(14):144110, 2010.

[101] Robin G E Kimber, Alison B Walker, Gerd E Schröder-Turk, and Douglas J Cleaver. Bicontinuous minimal surface nanostructures for polymer blend solar cells. Phys. Chem. Chem. Phys., 12(4):844$851,2010$.

[102] C Groves. Simulating charge transport in organic semiconductors and devices: a review. Reports Prog. Phys., 80(2):026502, feb 2017.

[103] Jean-Luc Brédas, David Beljonne, Veaceslav Coropceanu, and Jérôme Cornil. Charge-Transfer and Energy-Transfer Processes in $\pi$-Conjugated Oligomers and Polymers: A Molecular Picture. Chem. Rev., 104(11):4971-5004, nov 2004.

[104] Yi-kang Lan and Ching-i Huang. A Theoretical Study of the Charge Transfer Behavior of the Highly Regioregular Poly-3-hexylthiophene in the Ordered State. J. Phys. Chem. B, 112(47):14857-14862, nov 2008.

[105] R. a. Marcus. On the Theory of Oxidation-Reduction Reactions Involving Electron Transfer. I. J. Chem. Phys., 24(5):966, 1956.

[106] N. S. Hush. Adiabatic Rate Processes at Electrodes. I. Energy-Charge Relationships. J. Chem. Phys., 28(5):962, 1958.

[107] Attila J Mozer and Niyazi Serdar Sariciftci. Negative Electric Field Dependence of Charge Carrier Drift Mobility in Conjugated, Semiconducting Polymers. Chem. Phys. Lett., 389(4-6):438-442, may 2004.

[108] Amy M. Ballantyne, Lichun Chen, Justin Dane, Thomas Hammant, Felix M Braun, Martin Heeney, Warren Duffy, Iain McCulloch, Donal D C Bradley, and Jenny Nelson. The Effect of Poly(3hexylthiophene) Molecular Weight on Charge Transport and the Performance of Polymer:Fullerene Solar Cells. Adv. Funct. Mater., 18(16):2373-2380, aug 2008.

[109] Nenad Vukmirovic and Lin-Wang Wang. Electronic Structure of Disordered Conjugated Polymers: Polythiophenes. J. Phys. Chem. B, 113(2):409-415, jan 2009.

[110] Ralf Mauer, Marcel Kastler, and Frédéric Laquai. The Impact of Polymer Regioregularity on Charge Transport and Efficiency of P3HT:PCBM Photovoltaic Devices. Adv. Funct. Mater., 20(13):2085-2092, may 2010.

[111] T Koopmans. Über die Zuordnung von Wellenfunktionen und Eigenwerten zu den Einzelnen Elektronen Eines Atoms. Physica, 1(1-6):104-113, jan 1934.

[112] Veaceslav Coropceanu, Jérôme Cornil, Demetrio A da Silva Filho, Yoann Olivier, Robert Silbey, and Jean-Luc Brédas. Charge Transport in Organic Semiconductors. Chem. Rev., 107(4):926-952, apr 2007.

[113] J Ridley and Michael Zerner. An Intermediate Neglect of Differential Overlap Technique for Spectroscopy: Pyrrole and the Azines. Theor. Chim. Acta, 32(2):111-134, 1973.

[114] James Kirkpatrick. An Approximate Method for Calculating Transfer Integrals based on the ZINDO Hamiltonian. Int. J. Quantum Chem., 108(1):51-56, 2008.

[115] Vincent Lemaur, Demetrio a da Silva Filho, Veaceslav Coropceanu, Matthias Lehmann, Yves Geerts, Jorge Piris, Michael G Debije, Anick M van de Craats, Kittusamy Senthilkumar, Laurens D a Siebbeles, 
John M Warman, Jean-Luc Brédas, and Jérôme Cornil. Charge Transport Properties in Discotic Liquid Crystals: A Quantum-Chemical Insight into StructureProperty Relationships. J. Am. Chem. Soc., 126(10):3271-3279, mar 2004.

[116] Christine Corinne Mattheus. Polymorphism and Electronic Properties of Pentacene. PhD thesis, University of Groningen, 2002.

[117] Tao Liu and Alessandro Troisi. Absolute Rate of Charge Separation and Recombination in a Molecular Model of the P3HT/PCBM Interface. J. Phys. Chem. C, 115(5):2406-2415, feb 2011.

[118] Erik Johansson and Sven Larsson. Electronic Structure and Mechanism for Conductivity in Thiophene Oligomers and Regioregular Polymer. Synth. Met., 144(2):183-191, jul 2004.

[119] Tao Liu, David L Cheung, and Alessandro Troisi. Structural variability and dynamics of the P3HT/PCBM interface and its effects on the electronic structure and the charge-transfer rates in solar cells. Phys. Chem. Chem. Phys., 13(48):21461, 2011.

[120] Chris Groves. Suppression of Geminate Charge Recombination in Organic Photovoltaic Devices with a Cascaded Energy Heterojunction. Energy Environ. Sci., 6(5):1546, 2013.

[121] Matthew L Jones, Buddhapriya Chakrabarti, and Chris Groves. Monte Carlo Simulation of Geminate Pair Recombination Dynamics in Organic Photovoltaic Devices: Multi-Exponential, Field-Dependent Kinetics and Its Interpretation. J. Phys. Chem. C, 118(1):85-91, jan 2014.

[122] Manuel Schrader, Roland Fitzner, Moritz Hein, Chris Elschner, Björn Baumeier, Karl Leo, Moritz Riede, Peter Bäuerle, and Denis Andrienko. Comparative Study of Microscopic Charge Dynamics in Crystalline Acceptor-Substituted Oligothiophenes. J. Am. Chem. Soc., 134(13):6052-6056, apr 2012.

[123] Carl Poelking and Denis Andrienko. Effect of Polymorphism, Regioregularity and Paracrystallinity on Charge Transport in Poly(3-hexylthiophene) [P3HT] Nanofibers. Macromolecules, 46(22):8941-8956, nov 2013.

[124] Michael C. Heiber and Ali Dhinojwala. Efficient Generation of Model Bulk Heterojunction Morphologies for Organic Photovoltaic Device Modeling. Phys. Rev. Appl., 2(1):014008, jul 2014.

[125] H. Bässler. Charge Transport in Disordered Organic Photoconductors a Monte Carlo Simulation Study. Phys. Status Solidi B, 175(1):15-56, jan 1993.

[126] Stavros Athanasopoulos, James Kirkpatrick, Diego Martínez, Jarvist M Frost, Clare M Foden, Alison B Walker, and Jenny Nelson. Predictive Study of Charge Transport in Disordered Semiconducting Polymers. Nano Lett., 7(6):1785-1788, jun 2007.

[127] J C Blakesley, H S Clubb, and N C Greenham. Temperature-dependent electron and hole transport in disordered semiconducting polymers: Analysis of energetic disorder. Phys. Rev. B, 81(4):045210, jan 2010.

[128] W F Pasveer, J Cottaar, C Tanase, R Coehoorn, P A Bobbert, P W M Blom, D. M. de Leeuw, and M A J Michels. Unified Description of Charge-Carrier Mobilities in Disordered Semiconducting Polymers. Phys. Rev. Lett., 94(20):206601, may 2005.

[129] Artem A Bakulin, Akshay Rao, Vlad G. Pavelyev, P. H. M. van Loosdrecht, M. S. Pshenichnikov, Dorota Niedzialek, Jérôme Cornil, David Beljonne, and Richard H. Friend. The Role of Driving Energy and Delocalized States for Charge Separation in Organic Semiconductors. Science (80-. )., 335(6074):1340-1344, mar 2012.

[130] Askat E Jailaubekov, Adam P Willard, John R Tritsch, Wai-lun Chan, Na Sai, Raluca Gearba, Loren G Kaake, Kenrick J Williams, Kevin Leung, Peter J Rossky, and X-y Zhu. Hot Charge-Transfer Excitons Set the Time Limit for Charge Separation at Donor/Acceptor Interfaces in Organic Photovoltaics. Nat. Mater., 12(1):66-73, dec 2012.

[131] Paola Carbone and Alessandro Troisi. Charge Diffusion in Semiconducting Polymers: Analytical Relation between Polymer Rigidity and Time Scales for Intrachain and Interchain Hopping. J. Phys. Chem. Lett., 5(15):2637-2641, aug 2014.

[132] K. S. Haber and A C Albrecht. Time-of-Flight Technique for Mobility Measurements in the Condensed Phase. J. Phys. Chem., 88(24):6025-6030, nov 1984.

[133] Baijun Chen, Chun-sing Lee, Shuit-tong Lee, Patrick Webb, Yan-cheong Chan, William Gambling, He Tian, and Weihong Zhu. Improved Time-of-Flight Technique for Measuring Carrier Mobility in Thin Films of Organic Electroluminescent Materials. Jpn. J. Appl. Phys., 39(Part 1, No. 3A):11901192, mar 2000.

[134] R Dost, A Das, and M. Gruel. Time-of-Flight Mobility Measurements in Organic Field-Effect Transistors. J. Appl. Phys., 104(8):084519, 2008. 
[135] R.J. Kline, M.D. McGehee, E.N. Kadnikova, Jinsong Liu, and J.M.J. Fréchet. Controlling the FieldEffect Mobility of Regioregular Polythiophene by Changing the Molecular Weight. Adv. Mater., 15(18):1519-1522, sep 2003.

[136] Jean-marie Verilhac, Rafal Pokrop, Gilles LeBlevennec, Irena Kulszewicz-Bajer, Katarzyna Buga, Malgorzata Zagorska, Said Sadki, and Adam Pron. Molecular Weight Dependent Charge Carrier Mobility in Poly(3,3"-dioctyl-2,2':5',2"-terthiophene). J. Phys. Chem. B, 110(27):13305-13309, jul 2006.

[137] Shyam S. Pandey, Wataru Takashima, Shuichi Nagamatsu, Takeshi Endo, Masahiro Rikukawa, and Keiichi Kaneto. Regioregularity vs Regiorandomness: Effect on Photocarrier Transport in Poly (3hexylthiophene). Jpn. J. Appl. Phys., 39(Part 2, No. 2A):L94-L97, feb 2000.

[138] Youngkyoo Kim, Steffan Cook, Sachetan M. Tuladhar, Stelios A. Choulis, Jenny Nelson, James R. Durrant, Donal D. C. Bradley, Mark Giles, Iain McCulloch, Chang-Sik Ha, and Moonhor Ree. A Strong Regioregularity Effect in Self-Organizing Conjugated Polymer Films and High-Efficiency Polythiophene:Fullerene Solar Cells. Nat. Mater., 5(3):197-203, mar 2006.

[139] H. Sirringhaus, P. J. Brown, R. H. Friend, M. M. Nielsen, K. Bechgaard, B. M. W. Langeveld-Voss, a. J. H. Spiering, R. a. J. Janssen, E. W. Meijer, P. Herwig, and D. M. de Leeuw. Two-Dimensional Charge Transport in Self-Organized, High-Mobility Conjugated Polymers. Nature, 401(6754):685-688, oct 1999 .

[140] A Zen, J Pflaum, S Hirschmann, W Zhuang, F Jaiser, U. Asawapirom, J. P. Rabe, U Scherf, and D Neher. Effect of Molecular Weight and Annealing of Poly(3-hexylthiophene)s on the Performance of Organic Field-Effect Transistors. Adv. Funct. Mater., 14(8):757-764, aug 2004.

[141] Yi-Kang Lan and Ching-I Huang. Charge Mobility and Transport Behavior in the Ordered and Disordered States of the Regioregular Poly(3-hexylthiophene). J. Phys. Chem. B, 113(44):1455514564, nov 2009.

[142] Avishek R. Aiyar, Jung-Il Hong, Rakesh Nambiar, David M. Collard, and Elsa Reichmanis. Tunable Crystallinity in Regioregular Poly(3-Hexylthiophene) Thin Films and Its Impact on Field Effect Mobility. Adv. Funct. Mater., 21(14):2652-2659, jul 2011.

[143] Zhenan Bao, Ananth Dodabalapur, and Andrew J Lovinger. Soluble and Processable Regioregular poly(3-hexylthiophene) for Thin Film Field-Effect Transistor Applications with High Mobility. Appl. Phys. Lett., 69(26):4108, 1996.

[144] Henning Sirringhaus. Integrated Optoelectronic Devices Based on Conjugated Polymers. Science (80-. )., 280(5370):1741-1744, jun 1998.

[145] J. L. Bredas, J P Calbert, D A da Silva Filho, and J Cornil. Organic Semiconductors: A Theoretical Characterization of the Basic Parameters Governing Charge Transport. Proc. Natl. Acad. Sci., 99(9):5804-5809, apr 2002.

[146] Begoña Milián Medina, Antoine Van Vooren, Patrick Brocorens, Johannes Gierschner, Maxim Shkunov, Martin Heeney, Iain McCulloch, Roberto Lazzaroni, and Jérôme Cornil. Electronic Structure and Charge-Transport Properties of Polythiophene Chains Containing Thienothiophene Units: A Joint Experimental and Theoretical Study. Chem. Mater., 19(20):4949-4956, oct 2007.

[147] Alessandro Troisi, Giorgio Orlandi, and John E Anthony. Electronic Interactions and Thermal Disorder in Molecular Crystals Containing Cofacial Pentacene Units. Chem. Mater., 17(20):5024-5031, oct 2005.

[148] David L Cheung, David P McMahon, and Alessandro Troisi. Computational Study of the Structure and Charge-Transfer Parameters in Low-Molecular-Mass P3HT. J. Phys. Chem. B, 113(28):9393-9401, jul 2009.

[149] A Troisi. Prediction of the Absolute Charge Mobility of Molecular Semiconductors: the Case of Rubrene. Adv. Mater., 19(15):2000-2004, aug 2007.

[150] James Kirkpatrick, Valentina Marcon, Jenny Nelson, Kurt Kremer, and Denis Andrienko. Charge Mobility of Discotic Mesophases: A Multiscale Quantum and Classical Study. Phys. Rev. Lett., 98(22):227402, may 2007.

[151] Thorsten Vehoff, Bjorn Baumeier, Alessandro Troisi, and Denis Andrienko. Charge Transport in Organic Crystals: Role of Disorder and Topological Connectivity. J. Am. Chem. Soc., 132(33):1170211708, aug 2010.

[152] Linjun Wang, Qikai Li, Zhigang Shuai, Liping Chen, and Qiang Shi. Multiscale study of charge mobility of organic semiconductor with dynamic disorders. Phys. Chem. Chem. Phys., 12(13):3309, 2010 . 
[153] Olga Wodo, Srikanta Tirthapura, Sumit Chaudhary, and Baskar Ganapathysubramanian. A GraphBased Formulation for Computational Characterization of Bulk Heterojunction Morphology. Org. Electron., 13(6):1105-1113, jun 2012.

[154] Olga Wodo, John D. Roehling, Adam J. Moulé, and Baskar Ganapathysubramanian. Quantifying Organic Solar Cell Morphology: a Computational Study of Three-Dimensional Maps. Energy Environ. Sci., 6(10):3060, 2013.

[155] John Towns, Timothy Cockerill, Maytal Dahan, Ian Foster, Kelly Gaither, Andrew Grimshaw, Victor Hazlewood, Scott Lathrop, Dave Lifka, Gregory D. Peterson, Ralph Roskies, J. Ray Scott, and Nancy Wilkens-Diehr. XSEDE: Accelerating Scientific Discovery. Comput. Sci. Eng., 16(5):62-74, sep 2014. 